\title{
MODELING AND PERFORMANCE ANALYSIS OF PUBLIC SAFETY WIRELESS NETWORKS
}

\author{
by \\ Jiaqing Song \\ B.Eng., Soochow University, China, 2000 \\ THESIS SUBMITTED IN PARTIAL FULFILLMENT OF
THE REQUIREMENTS FOR THE DEGREE OF \\ MASTER OF APPLIED SCIENCE \\ In the \\ School \\ of \\ Computing Science \\ (C) Jiaqing Song 2005 \\ SIMON FRASER UNIVERSITY
}

Spring 2005

\footnotetext{
All rights reserved. This work may not be reproduced in whole or in part, by photocopy or other means, without permission of the author.
} 


\section{APPROVAL}

Name:

Degree:

Title of Thesis:

\section{Examining Committee:}

Chair:

\section{Dr. Jian Pei}

Assistant Professor of School of Computing Science

Dr. Ljiljana Trajković

Senior Supervisor

Professor of School of Engineering Science

Dr. Qianping Gu

Supervisor

Professor of School of Computing Science

Dr. Uwe Glässer

Internal Examiner

Associate Professor of School of Computing Science

Date Defended: 


\begin{abstract}
Public safety wireless networks (PSWNs) play a vital role in operations of emergency agencies such as police and fire departments. In this thesis, we describe analysis and modeling of traffic data collected from the Emergency Communications for Southwestern British Columbia (E-Comm) PSWN.

We analyze network and agency call traffic and find that lognormal distribution and exponential distribution are adequate for modeling call holding time and call interarrival time, respectively. We also describe a newly developed wide area radio network simulator, named WarnSim. We use WarnSim simulations to validate the proposed traffic model, evaluate the performance of the E-Comm network, and predict network performance in cases of traffic increase. We also provide recommendations on allocating E-Comm network resources to deal with the increased traffic volume.
\end{abstract}




\section{DEDICATION}

To my father Kejun Jia, my mother Shen'an Song, and my wife Zhen Yuan. 


\section{ACKNOWLEDGMENTS}

There are few things in the world that a person could accomplish alone. Writing this thesis is certainly not one of them. I would like to express my special thanks to my senior supervisor Dr. Ljiljana Trajković for the continuously guidance and everlasting support she has been giving me throughout my graduate studies.

My sincere gratitude goes to Dr. Qianping Gu, for being my supervisor and being my advising voice on my thesis. Furthermore, I would like to express appreciation to Dr. Uwe Glässer and Dr. Jian Pei for being on my examining committee and for giving me valuable suggestions on my thesis.

Finally, I would like to thank all members of the Communication Networks Laboratory at Simon Fraser University for numerous and enlightening discussions. 


\section{TABLE OF CONTENTS}

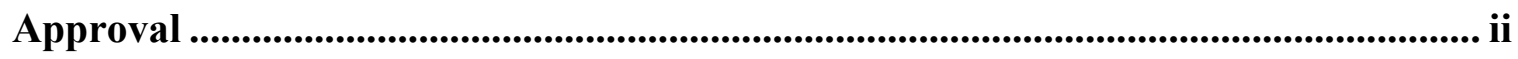

Abstract.............................................................................................................................. iii

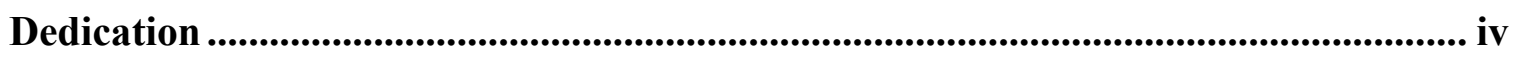

Acknowledgments ........................................................................................................

Table of Contents .......................................................................................................................... vi

List of Figures.................................................................................................................................. viii

List of Tables ........................................................................................................ ix

Glossary ..............................................................................................................................

Chapter 1: Introduction ..................................................................................................1

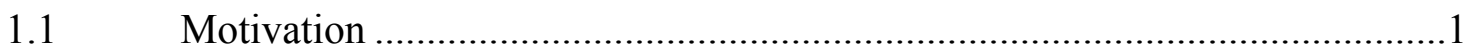

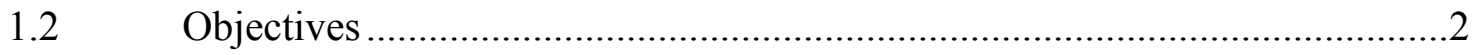

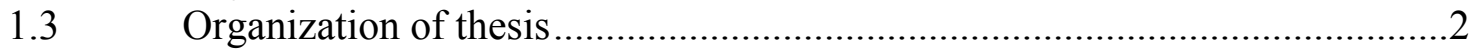

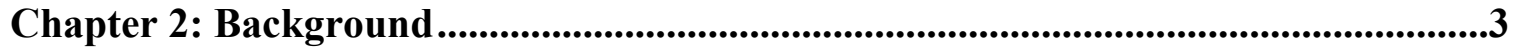

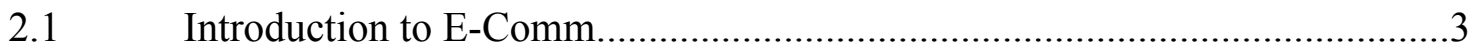

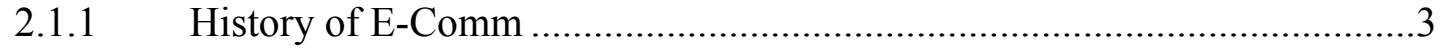

2.1.2 E-Comm network architecture...........................................................

2.1.3 Simulcast system .............................................................................

2.1.4 Transmission trunking and message trunking ............................................

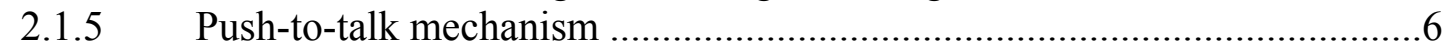

2.1.6 Talk group and group calls ...................................................................6

2.1.7 Systems and channels of the E-Comm network .......................................

2.1.8 Mobility of radio devices and call handover ...........................................

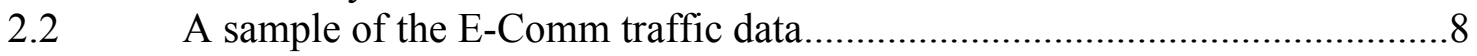

Chapter 3: Traffic data analysis.......................................................................................11

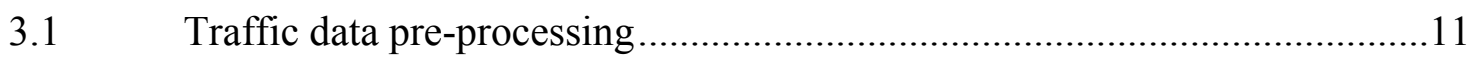

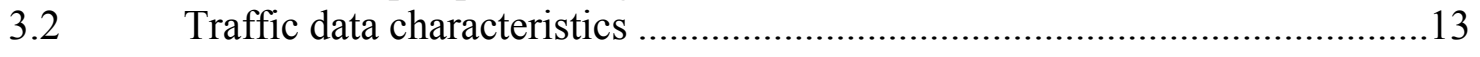

3.2.1 Hourly and daily call arrival rates .......................................................13

3.2.2 Call holding time ………..................................................................

3.2.3 Agencies and call arrival rates............................................................. 18

3.2.4 Systems and call arrival rates .............................................................19

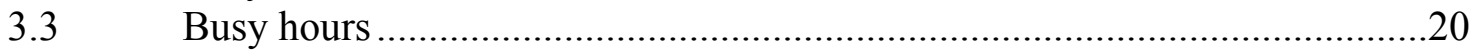

Chapter 4: Statistical traffic modeling............................................................................22

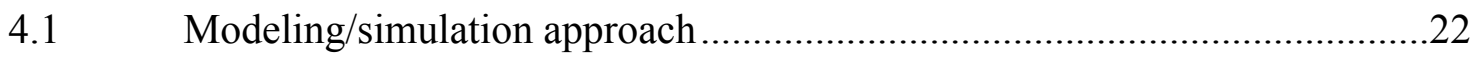


4.2 Introduction to traditional traffic models ...............................................23

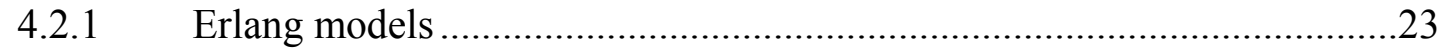

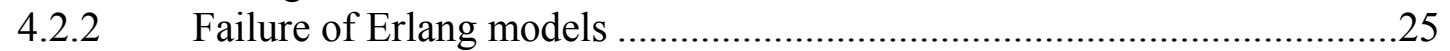

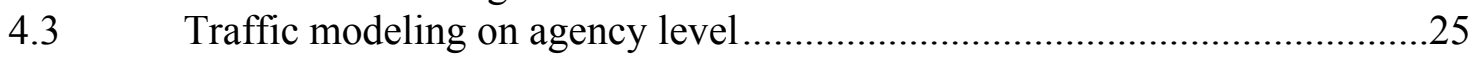

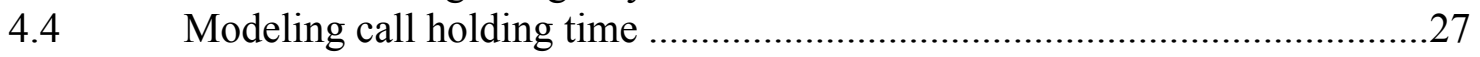

4.4.1 Maximum likelihood estimation.......................................................27

4.4.2 Kolmogorov-Smirnov goodness-of-fit test..........................................28

4.4.3 Survey of existing models for call holding time.....................................29

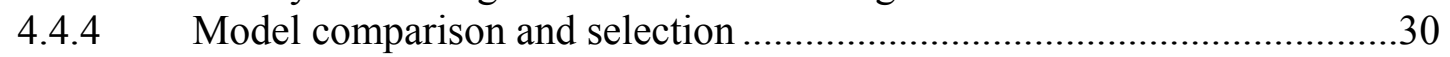

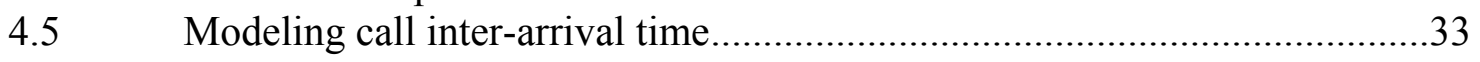

4.5.1 Survey of existing models for call inter-arrival time...............................33

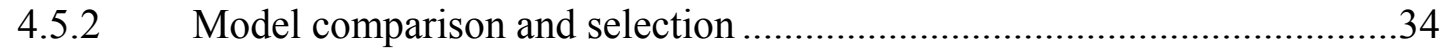

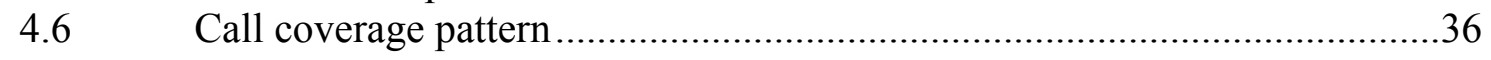

Chapter 5: WarnSim: a Simulator for PSWN ........................................................37

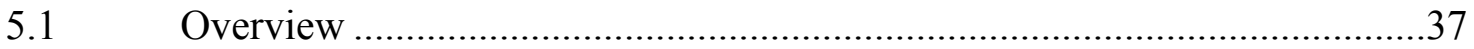

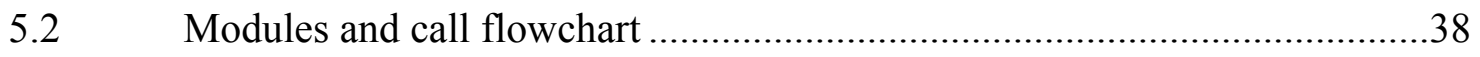

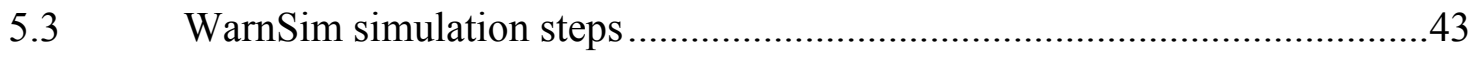

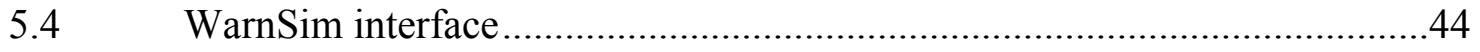

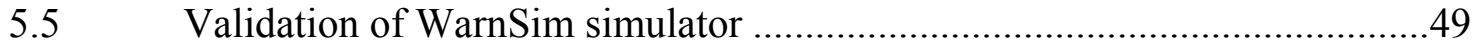

S.6 Scalability and performance of WarnSim ................................................50

Chapter 6: Simulation and prediction .................................................................51

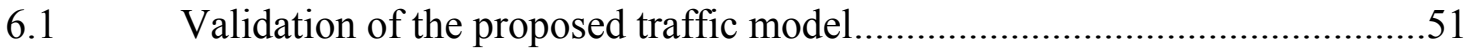

6.2 Evaluation of E-Comm network performance .........................................52

6.2.1 Network performance during busy hours ............................................52

6.2.2 Number of channels and Grade of Service ...............................................55

6.2.3 Maximum queuing time and Grade of Service........................................57

6.3 Prediction of the E-Comm network performance ...................................59

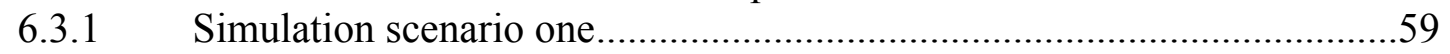

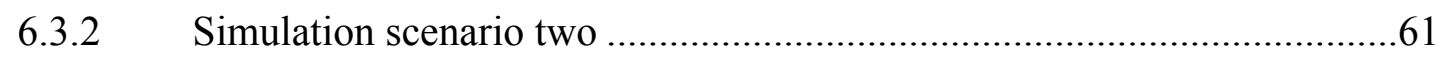

Chapter 7: Conclusions and future work ............................................................63

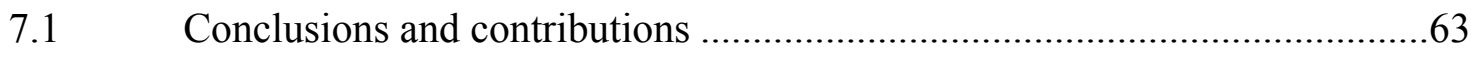

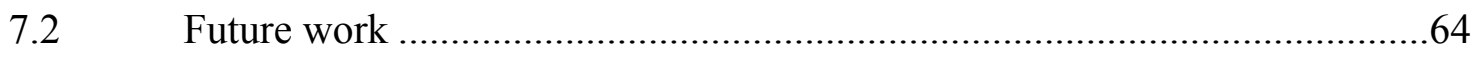

Appendix A: Maximum likelihood estimation (mle) scripts ........................................65

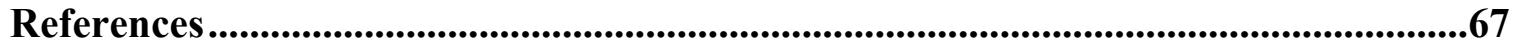




\section{LIST OF FIGURES}

Figure 2.1: System architecture of EDACS...........................................................4

Figure 3.1: Plot of the hourly call arrival rate over 30 days in year 2002 .......................13

Figure 3.2: Plot of the hourly call arrival rate over 92 days in year 2003. .......................14

Figure 3.3: Power spectrum of hourly call arrival from the 2003 dataset indicates a strong daily cyclic pattern. ............................................................... 15

Figure 3.4: Power spectrum of daily call arrival from the 2003 dataset indicates a

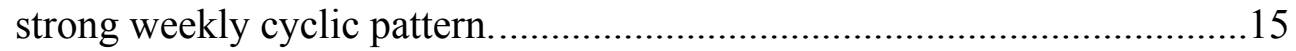

Figure 3.5: Average hourly call arrival rate over 24 hours...........................................16

Figure 3.6: Average daily call arrival rate over seven days..........................................17

Figure 3.7: Hourly average of call holding time over 92 days in year 2003. ...................17

Figure 3.8: Power spectrum of average hourly call holding time indicates a

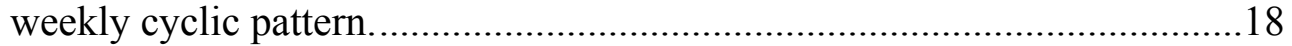

Figure 5.1: WarnSim: (a) module hierarchical diagram and (b) relationship

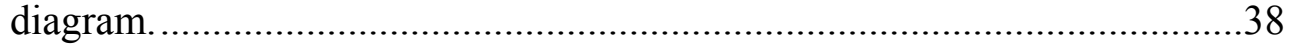

Figure 5.2: High level diagram of the WarnSim simulator. ........................................41

Figure 5.3: Pseudo-code corresponding to the high level diagram of the WarnSim

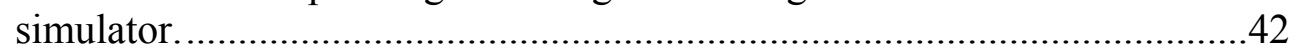

Figure 5.4: WarnSim: (a) network topology and (b) network configuration screen. .......45

Figure 5.5: WarnSim traffic source configuration screen: importing traffic. ...................46

Figure 5.6: WarnSim traffic source configuration screen: generating traffic...................46

Figure 5.7: WarnSim simulation results screens for channel utilization of Vancouver system during: (a) a sample week in 2002 and (b) a sample week in 2003. The graphs show the running average calculated over ten-minute intervals.

Figure 5.8: WarnSim simulation results screens for the cumulative number of blocked calls in Vancouver system during: (a) a sample week in 2002 and (b) a sample week in 2003.

Figure 6.1: Relationship between number of channels, call blocking probability, and channel utilization. Maximum queuing time is set to zero.

Figure 6.2: Maximum call queuing time vs. call blocking probability 


\section{LIST OF TABLES}

Table 2.1: Number of channels deployed in each E-Comm system as of December 2003.

Table 2.2: A sample of raw traffic data collected from the E-Comm network.

Table 2.3: Descriptions of data fields in the E-Comm traffic data table..........................10

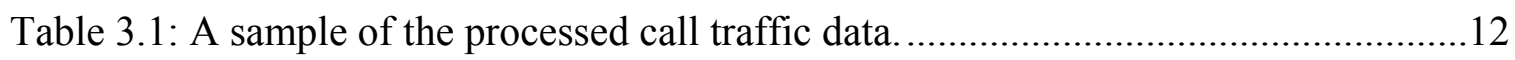

Table 3.2: Agencies and average daily call arrival rates. .............................................19

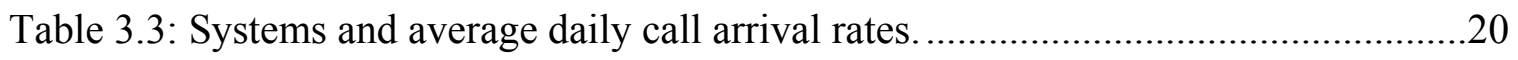

Table 3.4: The top three busiest hours in 2002 and 2003 traffic datasets.........................21

Table 4.1: Aggregated number of calls during the top three busiest hours in 2002

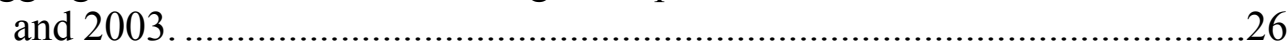

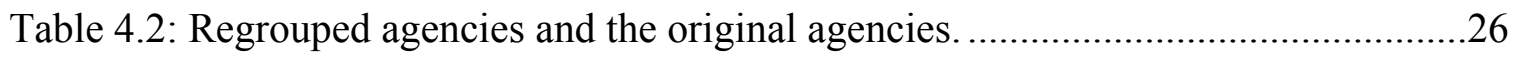

Table 4.3: Candidate distributions for call holding time. .................................................31

Table 4.4: Test of candidate distributions for call holding time of Agency A during busy hours in the 2002 dataset........................................................

Table 4.5: Test of candidate distributions for call holding time of Agency B during the top three busy hours in the 2002 dataset......................................32

Table 4.6: Test of candidate distributions for call holding time of Agency $\mathrm{C}$ during the top three busy hours in the 2002 dataset........................................33

Table 4.7: Candidate distributions for call inter-arrival time. ............................................34

Table 4.8: Test of candidate distributions for call inter-arrival time of Agency A during the top three busy hours in the 2002 dataset.......................................35

Table 4.9: Test of candidate distributions for call inter-arrival time of Agency B during the top three busy hours in the 2002 dataset........................................35

Table 4.10: Test of candidate distributions for call inter-arrival time of Agency C during the top three busy hours in the 2002 dataset......................................36

Table 4.11: A sample of call coverage pattern of Agency A during the top three busy hours in 2003.

Table 5.1: Comparison of call blocking probability predicted by Erlang B model and the WarnSim simulated call blocking probability.

Table 6.1: Distribution parameters for the WarnSim call generator....................................51

Table 6.2: System IDs and number of channels. .......................................................51

Table 6.3: Comparison of network performance: using collected traffic (actual) vs. model generated traffic (simulated). 
Table 6.4: System IDs and number of channels during the top three busy hours in the 2003 dataset.

Table 6.5: Simulation results of the busiest hour in the 2003 dataset...............................54

Table 6.6: Simulation results of the second busiest hour in the 2003 dataset. ..................54

Table 6.7: Simulation results of the third busiest hour in 2003 dataset............................55

Table 6.8: Relationship between number of channels, call blocking probability, and channel utilization. Maximum queuing time is set to zero.....................56

Table 6.9: Maximum call queuing time vs. call blocking probability................................58

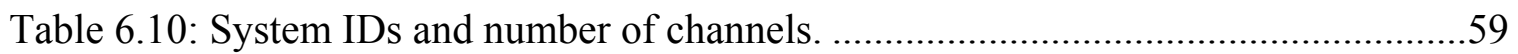

Table 6.11: Scenario one: distribution parameters for the WarnSim call generator..........60

Table 6.12: Comparison of network performance. Scenario one: using collected traffic (original) vs. model generated traffic after the increase (predicted). Cells with call blocking probability higher than $3 \%$ are marked in grey....

Table 6.13: Scenario two: distribution parameters for the WarnSim call generator. .........61

Table 6.14: Comparison of network performance. Scenario two: using collected traffic (original) vs. model generated traffic after the increase (predicted). Cells with call blocking probability higher than 3\% are marked in grey 


\section{GLOSSARY}

\begin{tabular}{|c|c|}
\hline $\mathrm{CDF}$ & Cumulative Density Function \\
\hline E-Comm & Emergency Communications for Southwestern British Columbia Inc. \\
\hline ECDF & Empirical Distribution Function \\
\hline EDACS & Enhanced Digital Access Communications System \\
\hline FIFO & First In First Out \\
\hline GoS & Grade of Service \\
\hline GVRD & Great Vancouver Regional District \\
\hline K-S GoF test & Kolmogorov-Smirnov Goodness-of-Fit Test \\
\hline LMR & Land Mobile Radio \\
\hline MLE & Maximum Likelihood Estimation \\
\hline PDF & Probability Density Function \\
\hline PAMR & Public Access Mobile Radio \\
\hline PCS & Personal Communication Services \\
\hline PMR & Private Mobile Radio System \\
\hline PSTN & Public Switched Telephone Network \\
\hline PSWN & Public Safety Wireless Network \\
\hline PTT & Push-to-Talk \\
\hline WARN & Wide Area Radio Network \\
\hline WarnSim & Wide Area Radio Network Simulator \\
\hline
\end{tabular}




\section{CHAPTER 1: INTRODUCTION}

\subsection{Motivation}

Sharing vital voice information via radio in a timely and reliable manner is critical for operations of various public safety agencies, such as law enforcement, fire departments, and emergency medical services. Hence, public safety wireless networks (PSWNs) employed for on-scene communications play an important role in ensuring public safety.

The public safety community has identified the limited and fragmented radio spectrum as one of the key issues that adversely affects public safety wireless communications [1]. In the United States, the SAFECOM program was recently established to serve as the umbrella program within the Federal Government to help local, state, and federal public safety agencies improve public safety response through more effective and efficient wireless communications [2]. The emergency wireless communication service providers are also concerned with the spectrum shortage and the high cost of deploying and operating voice channels. For example, emergency agencies covering Vancouver share only eleven wireless voice channels. Therefore, understanding, analyzing, evaluating, and optimizing public safety wireless networks are a valuable research topic.

We use a modeling/simulation approach and a customized simulator named WarnSim to evaluate and predict the performance of the public safety wireless network (PSWN) deployed in British Columbia, Canada. 


\subsection{Objectives}

The objectives of this project are to develop statistical models for call traffic in

the E-Comm network, to evaluate performance of the E-Comm network in terms of channel utilization and call blocking probability, and to predict future performance of the E-Comm network via a simulation approach.

\subsection{Organization of thesis}

This thesis is organized as follows. We introduce the structure of the E-Comm network in Chapter 2. Analysis of the E-Comm traffic data is presented in Chapter 3. In Chapter 4, we describe statistical modeling of call holding and call inter-arrival times, two components of a statistical model for call traffic. In Chapter 5, We introduce the design and implementation of a customized simulator for wide area radio networks, named WarnSim. Simulations performed with WarnSim and the simulation results are presented in Chapter 6. Conclusions and future work are given in Chapter 7. Scripts for Maximum Likelihood Estimation (MLE) are given in Appendix A. 


\section{CHAPTER 2: BACKGROUND}

In this Chapter, we introduce the history of E-Comm. We also describe the network architecture and the traffic data collected from the E-Comm network.

\subsection{Introduction to E-Comm}

\subsubsection{History of E-Comm}

On June $14^{\text {th }}, 1994$, the Stanley Cup riots took place in downtown Vancouver, Canada. Several emergency agencies, including Vancouver police, fire department, the RCMP, and BC Ambulance, were mobilized to handle the emergency situation. After the disturbance, it was discovered that communications between these emergency agencies were highly inadequate. One reason was that these emergency agencies operated their own wireless radio networks that were not interconnected. Moreover, emergency radio channels utilized by the police and fire departments needed significant capacity upgrading. To solve these problems, the government of British Columbia initiated a new emergency communication project. This project evolved into E-Comm in 1997.

E-Comm is the regional Emergency Communications Centre for Southwest British Columbia. E-Comm provides wide area radio dispatching services for a variety of emergency agencies throughout the Greater Vancouver Regional District (GVRD), the Sunshine Coast Regional District, Whistler, and Pemberton [3]. Until 2004, the E-Comm project has a total investment of $\$ 160$ million CAD and an annual operating budget of \$41 million CAD [4]. 


\subsubsection{E-Comm network architecture}

E-Comm's wireless radio network utilizes the Enhanced Digital Access Communications System (EDACS), manufactured by M/A-Com [5]. Its architecture is shown in Figure 2.1. It contains a central system controller (network switch), a management console, several repeater sites (base stations), one or more fixed user sites (dispatch consoles), a private branch exchange (PBX) gateway to the public switched telephone network (PSTN), and thousands of mobile users. Individual systems (cells) that cover separate geographic regions (City of Vancouver, City of Burnaby) in the E-Comm network are interconnected by high-speed data links. System events and call activities in the network are recorded by base stations and are forwarded every hour through the data gateway to the central database.

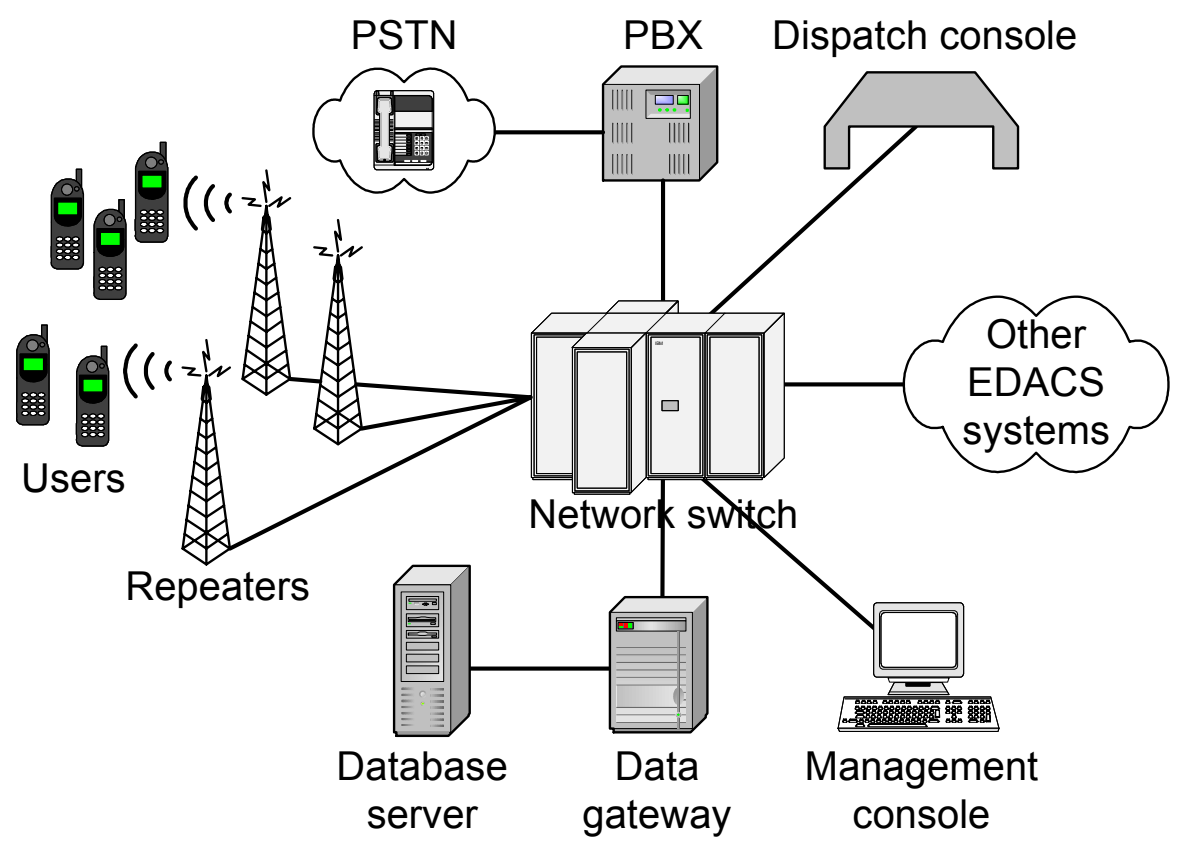

Figure 2.1: System architecture of EDACS. 


\subsubsection{Simulcast system}

EDACS may operate in one of the five modes: single site system, voted system, simulcast system, single channel system, and multi-site system. Systems (cells) in the EComm network are simulcast systems that employ multiple repeaters to transmit and receive identical audio and data information over the same carrier frequency. For example, there are five simulcast repeaters covering the City of Vancouver. Simulcast systems are employed when it is necessary to use a limited number of frequencies to cover an area too large for a single repeater. The simulcast system may also provide higher signal strength and better fault tolerance.

\subsubsection{Transmission trunking and message trunking}

Communications within the E-Comm network are conducted via transmission trunking rather than via traditional message trunking. With message trunking, a radio channel is assigned to a call for the entire duration of the conversation. Because gaps of silent periods usually exist during a conversation, occupying the channel during the entire conversation time is inefficient in terms of channel utilization. With transmission trunking, the radio channel is automatically released as soon as the caller completes the sentence and releases the push-to-talk (PTT) button. The next speaker may begin a call by pushing the PTT button on his/her radio device. The system then checks the channel availability and assigns required number of channels to the call. Transmission trunking is $20-25 \%$ more efficient than message trunking [5]. However, there is a price for the high channel utilization in the transmission trunking mode. Overheads due to channel assigning time and channel dropping time are added to each transmission because the processes of channel assigning and channel dropping are repeated for every press of the 
PTT button. The continuously available high-speed control channel in the E-Comm network alleviates this overhead problem. The control channel uses a dedicated radio channel that supports $9.6 \mathrm{kbps}$ digital signalling. The channel assigning and channel dropping times are 0.25 seconds and 0.16 seconds, respectively.

\subsubsection{Push-to-talk mechanism}

The communication mechanism in the E-Comm network is push-to-talk (PTT). It implies that a user who wishes to initiate a call needs to press a button on the radio device to request one or more channels. The central switch locates users in the specific talk group and checks the availability of channels in systems covering the talk group. The caller receives an audible signal if there are free channels to establish the call. Otherwise, the call request is queued.

\subsubsection{Talk group and group calls}

In the E-Comm network, talk groups are defined at various levels (agency level, fleet level, and sub-fleet level) for better coordination of operations. Each radio device belongs to one or more talk groups and may be switched between talk groups.

Group call is the standard call type in the E-Comm network. Call recipients are members of a talk group. The advantage of a group call is that it eliminates the need for radio users to know the target device number in order to reach an individual user. Users may call a target group without having to know the current members of the group.

It is important to note that in the E-Comm network, a single call might use several channels simultaneously. If all users of a talk group reside within a single system, the network controller will allocate one free channel to the call. However, if members of a 
talk group are distributed across several systems, the network controller will allocate to the call a free channel in each system.

\subsubsection{Systems and channels of the E-Comm network}

E-Comm has eleven systems deployed in the GVRD as of December 2003. Table 2.1 shows the system ID, system coverage, and number of deployed channels in each system.

Table 2.1: Number of channels deployed in each E-Comm system as of December 2003.

\begin{tabular}{|c|l|c|}
\hline System ID & Coverage & Number of channels \\
\hline 1 & Vancouver & 13 \\
\hline 2 & Burnaby & 9 \\
\hline 3 & Maple Ridge & 7 \\
\hline 4 & Langley & 6 \\
\hline 5 & Seymour & 6 \\
\hline 6 & Port Coquitlam & 8 \\
\hline 7 & Richmond & 7 \\
\hline 8 & Mission & 5 \\
\hline 9 & Surrey & 9 \\
\hline 10 & South Surrey & 8 \\
\hline 11 & Bowen Island & 4 \\
\hline
\end{tabular}

\subsubsection{Mobility of radio devices and call handover}

Mobility of radio devices and call handover are two major concerns for micro-cell cellular networks. However, they are of little importance in the E-Comm network. The EComm network is a wide area radio network with each system covering a citywide area. Because an emergency call lasts 3.8 seconds on average, there is only a negligible probability that one radio device moves between two systems during such a short time. 


\subsection{A sample of the E-Comm traffic data}

All call activities occurred in the E-Comm network are recorded by base stations and are stored in the central database. Thus, the central database is the ideal location for

collecting network wide traffic data that contain call activity information from all systems. Table 2.2 shows a sample of raw call traffic data collected from the E-Comm network. The 26 fields of the raw traffic data table are explained in Table 2.3. 
Table 2.2: A sample of raw traffic data collected from the E-Comm network.

\begin{tabular}{|c|c|c|c|c|}
\hline \multicolumn{2}{|c|}{ Event_UTC } & Duration_ms & Network_Id & Node_Id \\
\hline \multicolumn{2}{|c|}{$2003-05-01$ 00:00:09.620 } & 1990 & 1 & 33 \\
\hline \multicolumn{2}{|c|}{ 2003-05-01 00:00:10.020 } & 1990 & 1 & 33 \\
\hline \multicolumn{2}{|c|}{$2003-05-01$ 00:00:11.607 } & 1990 & 1 & 33 \\
\hline \multicolumn{2}{|c|}{ 2003-05-01 00:00:12.010 } & 1990 & 1 & 33 \\
\hline System_Id & Channel_Id & Slot_Id & Caller & Callee \\
\hline 7 & 4 & NULL & 9999 & 1111 \\
\hline 1 & 4 & NULL & 9999 & 1111 \\
\hline 7 & 4 & NULL & 9999 & 1111 \\
\hline 1 & 4 & NULL & 9999 & 1111 \\
\hline Call_Type & Call_State & Call_Direction & Interconnect_Call & Digital_Call \\
\hline 0 & 0 & 0 & 0 & 1 \\
\hline 0 & 0 & 0 & 0 & 1 \\
\hline 0 & 1 & 100 & 0 & 1 \\
\hline 0 & 1 & 100 & 0 & 1 \\
\hline Voice_Call & Mcp & Confirmed_Call & Msg_Trunked_Call & Preempt_Call \\
\hline 1 & 1 & 0 & 0 & 0 \\
\hline 1 & 1 & 0 & 0 & 0 \\
\hline 1 & 0 & 1 & 0 & 0 \\
\hline 1 & 0 & 1 & 0 & 0 \\
\hline Primary_Call & Queue_Depth & Queue_Pri & Multi_System_Call & Caller_Bill \\
\hline 1 & NULL & NULL & 0 & 0 \\
\hline 1 & NULL & NULL & 0 & 0 \\
\hline 1 & NULL & NULL & 0 & 0 \\
\hline 1 & NULL & NULL & 0 & 0 \\
\hline Callee_Bill & Reason_Code & & & \\
\hline 0 & 0 & & & \\
\hline 0 & 0 & & & \\
\hline 0 & 0 & & & \\
\hline 0 & 0 & & & \\
\hline
\end{tabular}


Table 2.3: Descriptions of data fields in the E-Comm traffic data table.

\begin{tabular}{|c|c|}
\hline \multicolumn{2}{|c|}{ Traffic data fields of interests in this thesis: } \\
\hline Event_UTC & Call arrival time \\
\hline Duration_ms & Call holding time (call duration) \\
\hline System_Id & System ID in which a call occurred \\
\hline Channel_Id & Channel ID in which a call was using \\
\hline Caller & ID of a radio device that initiates a call \\
\hline Callee & ID of a radio device that receives a call \\
\hline Queue_Depth & Number of calls waiting in the queue \\
\hline \multicolumn{2}{|c|}{ Traffic data fields that are not used in this thesis: } \\
\hline Network_Id & Constant “1” \\
\hline Node_Id & Constant “33" \\
\hline Slot_Id & Constant "NULL" \\
\hline Call_Type & $\begin{array}{l}\text { Call type (group/individual/emergency/group set/system } \\
\text { all/Morse code/test/paging/scramble data/sys login/start } \\
\text { emergency/cancel emergency) }\end{array}$ \\
\hline Call_State & $\begin{array}{l}\text { Call state (channel assign/channel drop/key/un-key/digits/over } \\
\text { digits/queue/busy/deny/convert to callee) }\end{array}$ \\
\hline Slot_Id & Constant "NULL" \\
\hline Call_Direction & Indicator of making or receiving a call \\
\hline Voice_Call & Indicate if the call contains voice information \\
\hline Digital_Call & Indicate if the call is from digital device or analog device \\
\hline Interconnect_Call & Indicate if it is call to PSTN \\
\hline Multi_System_Call & Indicate if it is a multi-system call \\
\hline Confirmed_Call & Indicate if it is a confirmed call \\
\hline Msg_Trunked_Call & Indicate if the call is message trunking or transmission trunking \\
\hline Preempt_Call & Indicate if it is a pre-empt call \\
\hline Primary_Call & Indicate if it is a real call or group set signal \\
\hline Queue_Pri & The priority of a call in a queue \\
\hline Mcp & Indicator of multiple channel partition \\
\hline Caller_Bill & Indicate who (caller or callee) will pay for the call \\
\hline Callee_Bill & Indicate who (callee or caller) will pay for the call \\
\hline Reason_Code & Additional information \\
\hline
\end{tabular}




\section{CHAPTER 3: TRAFFIC DATA ANALYSIS}

In this Chapter, we first describe the procedure for pre-processing the E-Comm raw traffic data. We then analyze the call traffic data and describe their characteristics. To illustrate the evolution of the E-Comm network, we also compare the E-Comm traffic data collected from year 2002 with the traffic data collected from year 2003.

\subsection{Traffic data pre-processing}

We analyze three sets of call traffic data from the E-Comm network. These sets contain (year-month-day) 2 days (2001-11-01 to 2001-11-02), 30 days (2002-02-09 to 2002-03-10), and 92 days (2003-03-01 to 2003-05-31) of call traffic data.

In the raw traffic data, one row represents one event that occurred in the E-Comm network. A single call usually generates two or more events. For example, if one call covers Systems 1, 2, and 3, it will generate a channel-assigning event and a channeldropping event in all three systems. Thus, this call generates six events in the E-Comm network and it is recorded in 6 rows in the raw traffic data table. For the sake of analysis, it is desirable to combine these duplicated rows and use only one row to represent one call. Furthermore, it is necessary to delete redundant and irrelevant system events from the raw traffic data table. We follow six steps to pre-process raw traffic data:

Step 1: Delete redundant and irrelevant data rows. Keep only rows that represent genuine voice calls. 
Step 2: Ignore columns (data fields) that are irrelevant to our research: Network_Id, Node_Id, Slot_Id, Interconnect_Call, Caller_Bill, Callee_Bill, Voice_Call, Msg_Trunked_Call, Reason_Code, Digital_Call, Primary_Call, Call_Direction, Multi_System_Call, Preempt_Call, Queue_Depth, and Confirmed_Call.

Step 3: Combine data rows that represent a single call into one row. Update fields Systems and Channels.

Step 4: Add a new field named Caller_Agency into the traffic data table.

Step 5: Retrieve caller agency ID information based on Caller ID and update field Caller_Agency.

Step 6: Sort calls by the timestamp field Event_UTC (call arrival time).

A sample of the processed traffic data corresponding to the raw data shown in Table 2.2 is shown in Table 3.1. The data row in Table 3.1 depicts a call made by caller 9999 to callee 1111 at 2003-05-01 00:00:09.620. The call lasted for 1,990 ms and covered Systems 1 and 7. The call employed Channels no. 3 and no. 4 in Systems 1 and 7, respectively. The caller belonged to Agency 5 .

Table 3.1: A sample of the processed call traffic data.

\begin{tabular}{|l|l|l|l|}
\hline \multicolumn{2}{|l|}{ Call arrival time } & Duration $(\mathrm{ms})$ & Caller agency \\
\hline $2003-05-01$ 00:00:09.620 & 1,990 & 5 \\
\hline Caller & Callee & System ID & Channel no. \\
\hline 9999 & 1111 & 1,7 & 3,4 \\
\hline
\end{tabular}




\subsection{Traffic data characteristics}

\subsubsection{Hourly and daily call arrival rates}

We plot the hourly call arrival rate of 2002 and 2003 traffic data to visually examine the pattern of the hourly call arrival rate (number of calls that arrived within one hour). Figures 3.1 and 3.2 show cyclic patterns of the hourly call arrival rates in 2002 and 2003 datasets, respectively.

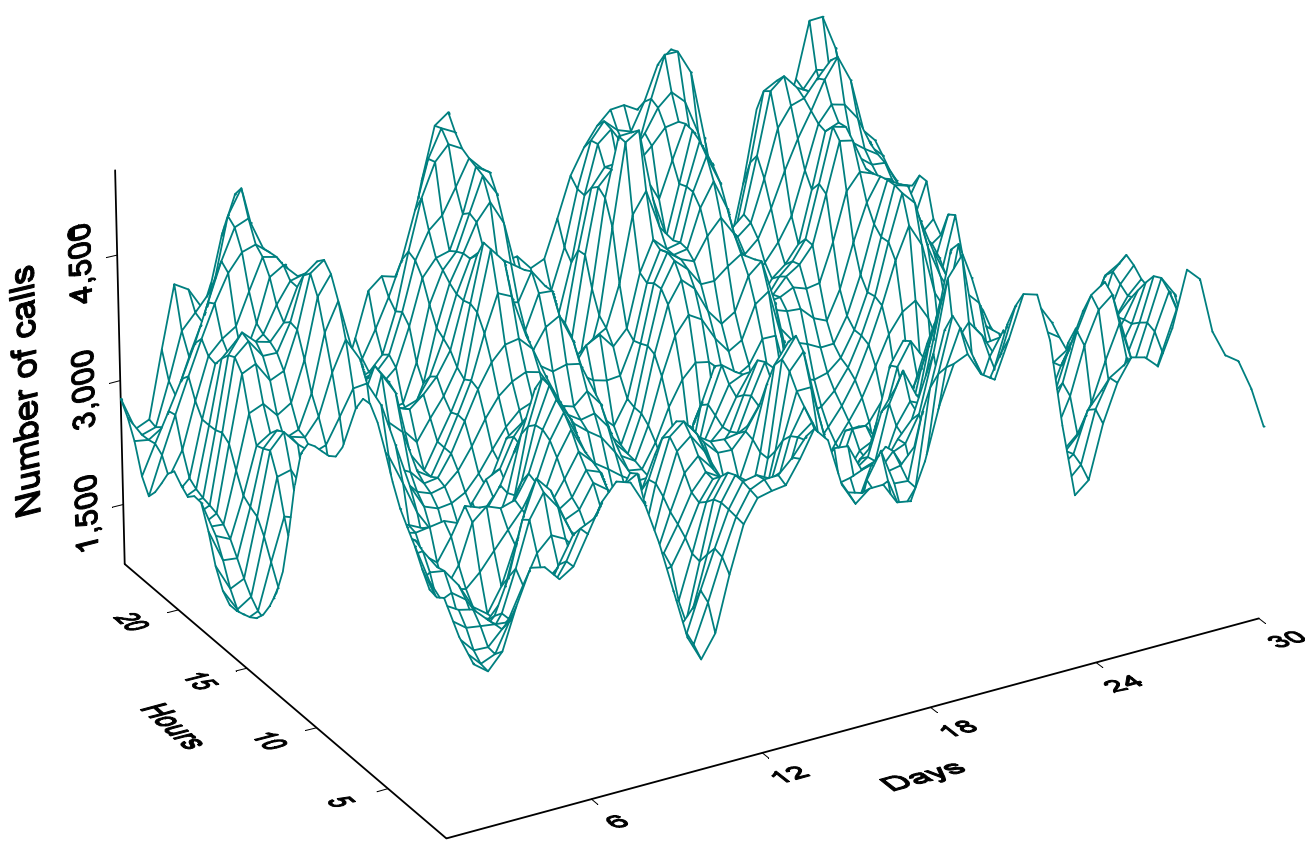

Figure 3.1: Plot of the hourly call arrival rate over 30 days in year 2002. 


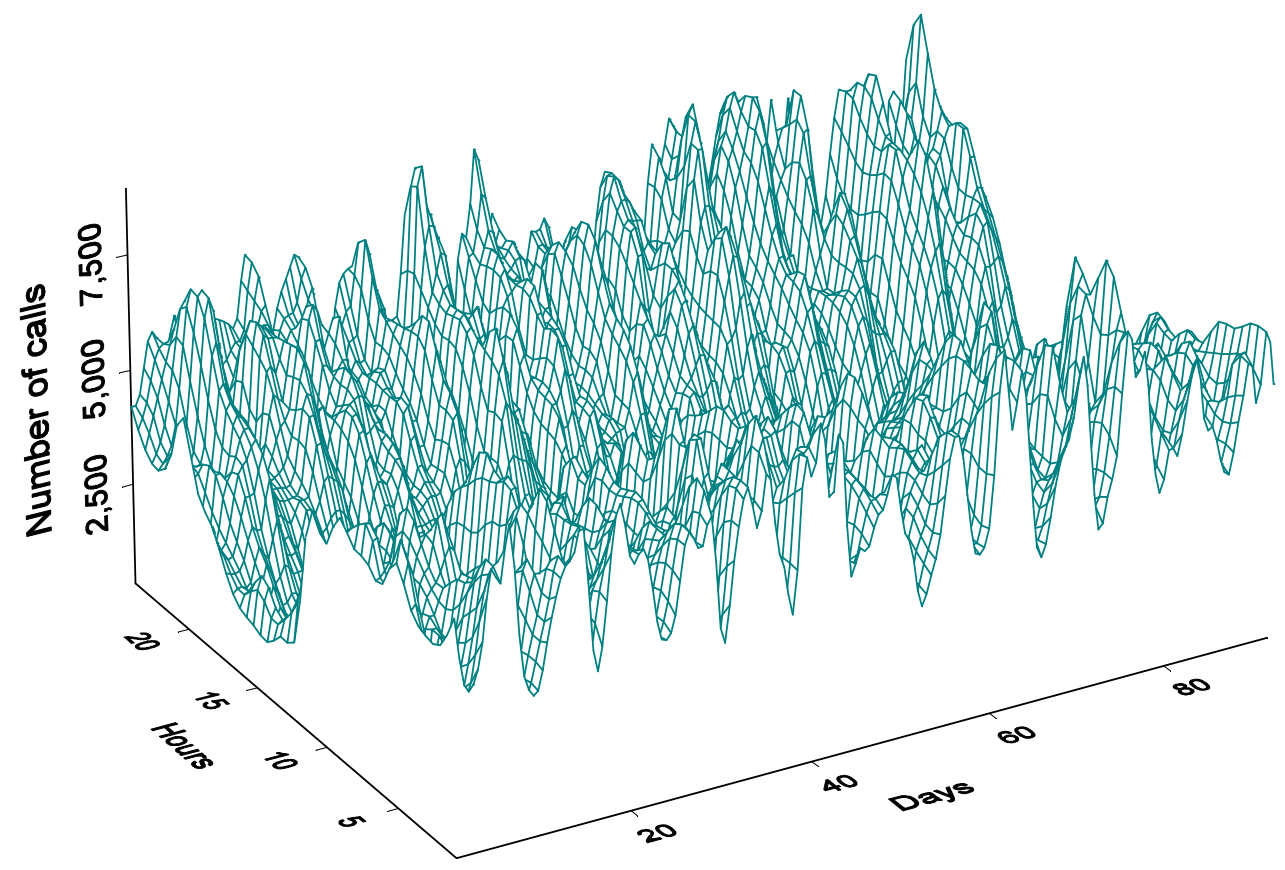

Figure 3.2: Plot of the hourly call arrival rate over 92 days in year 2003.

We consider 2,208 hours (92 days) of continuous traffic data in the 2003 dataset and use Fourier analysis to analyze hourly and daily call arrival rates in frequency domain [6]. The power spectrum of hourly and daily call arrivals are shown in Figures 3.3 and 3.4 , respectively. 


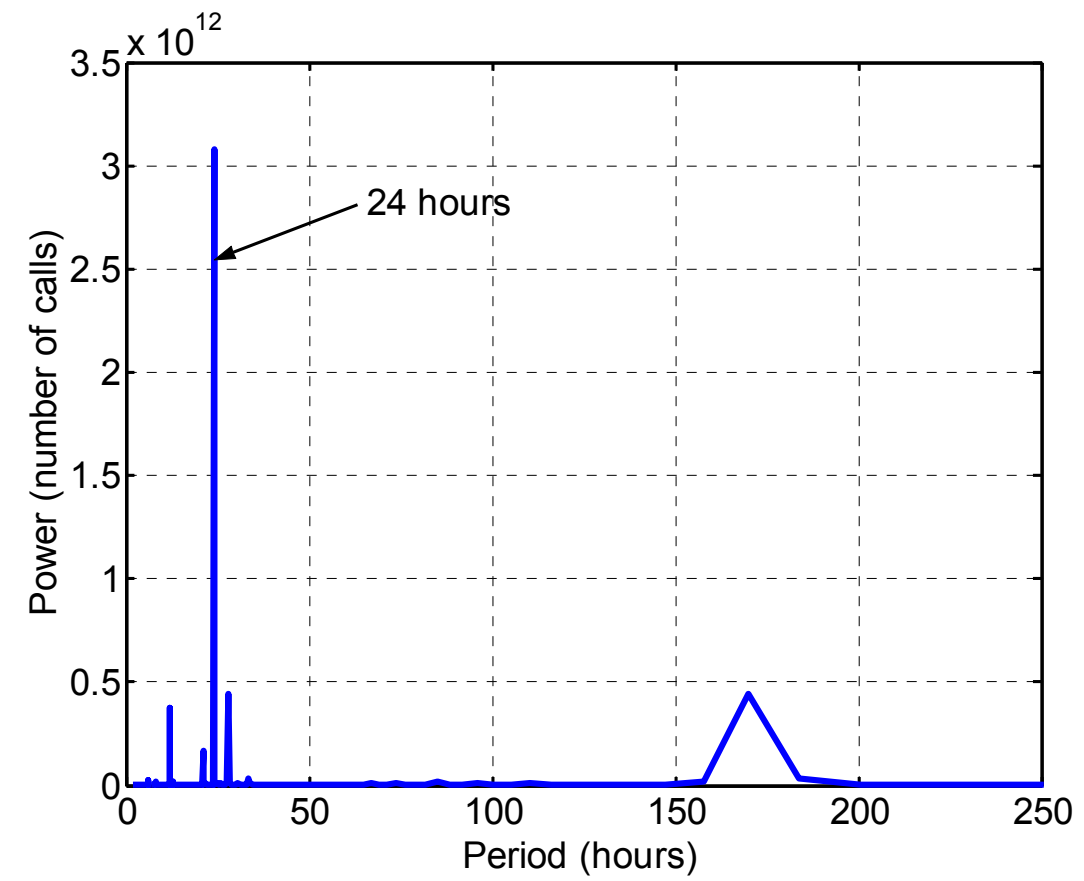

Figure 3.3: Power spectrum of hourly call arrival from the 2003 dataset indicates a strong daily cyclic pattern.

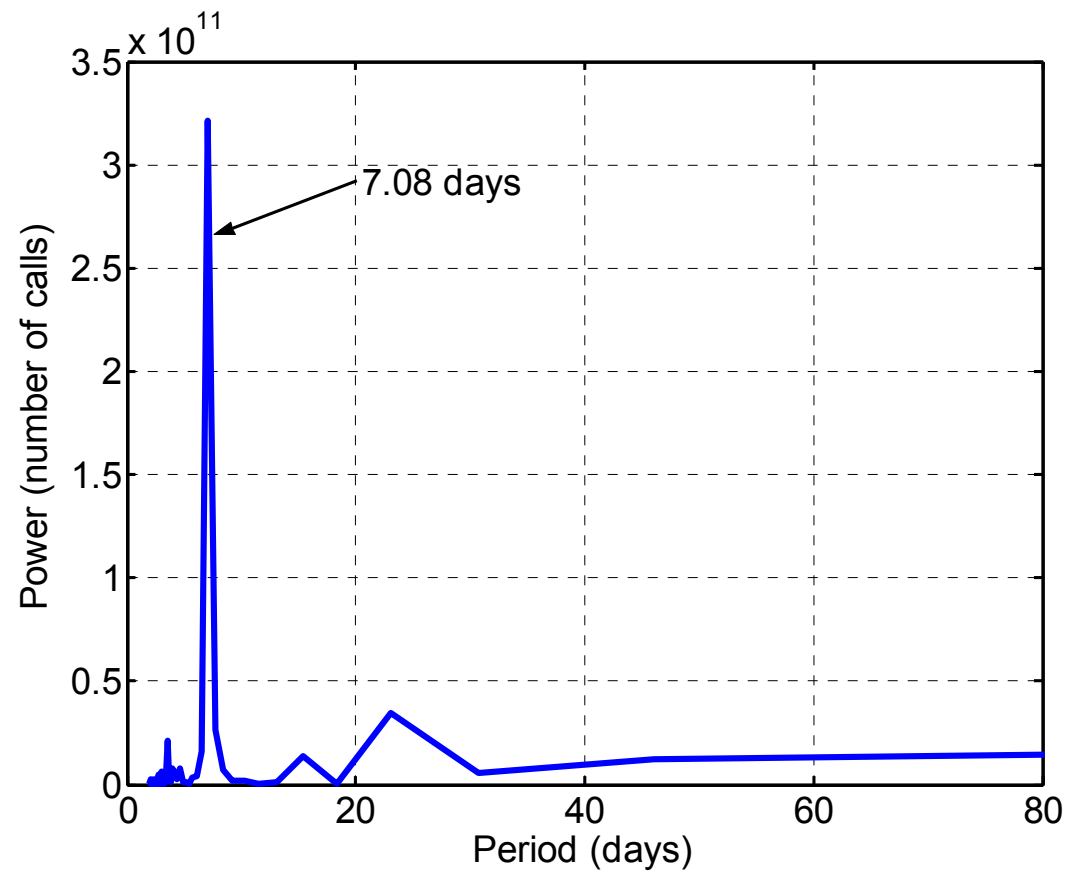

Figure 3.4: Power spectrum of daily call arrival from the 2003 dataset indicates a strong weekly cyclic pattern.

Further analysis results shown in Figure 3.5 indicate that the busiest hour in a day is around midnight, while the least amount of traffic is expected between $2 \mathrm{pm}$ and $3 \mathrm{pm}$. 
Furthermore, Thursday is the busiest day of a week, while Monday has the least number of calls, as shown in Figure 3.6.

The observations of the cyclic patterns of the call arrival rates are important because they provide useful guidelines for the E-Comm operators to schedule regular network maintenance tasks, such as database backup, to be performed when the network has least traffic.

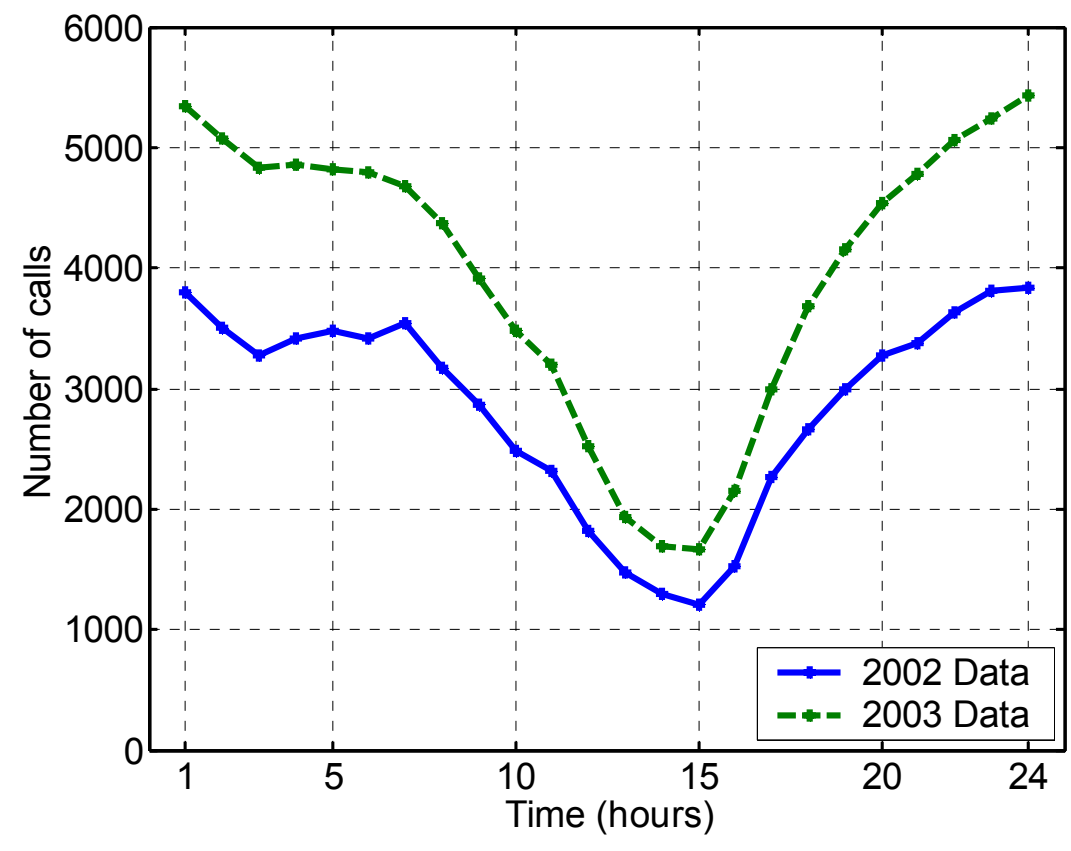

Figure 3.5: Average hourly call arrival rate over 24 hours. 


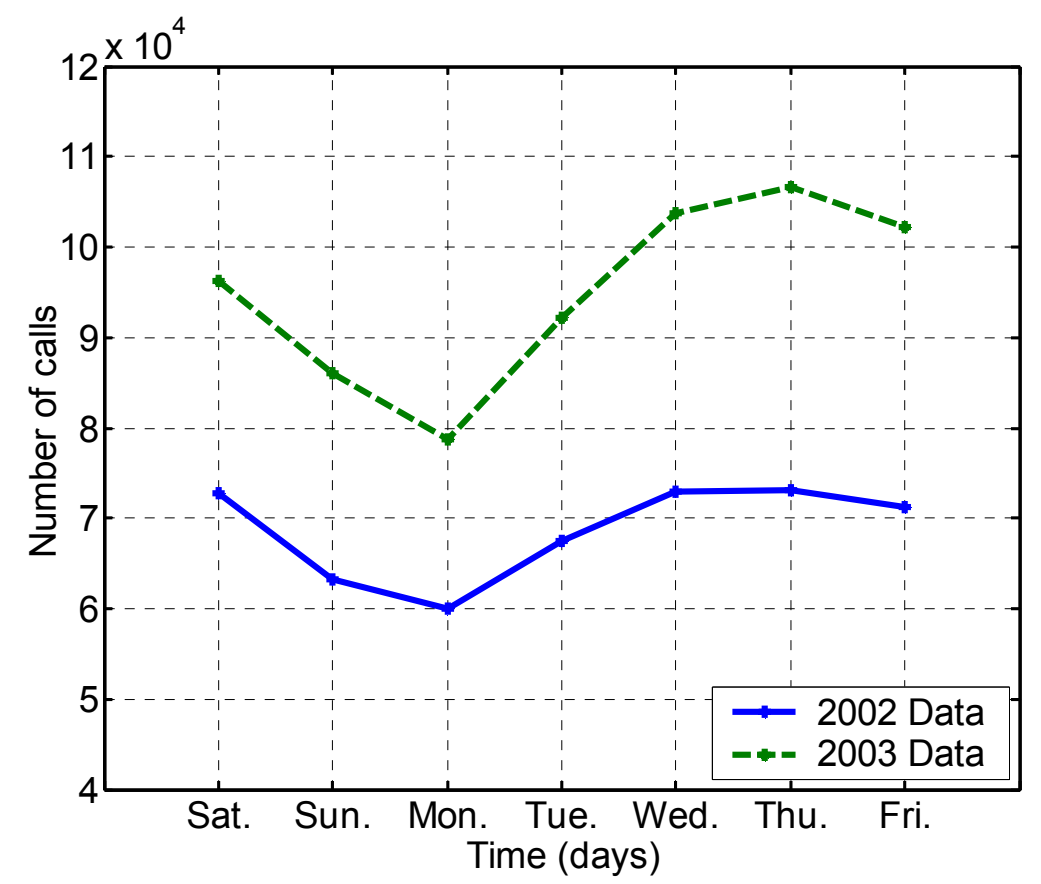

Figure 3.6: Average daily call arrival rate over seven days.

\subsubsection{Call holding time}

We also analyze the hourly average of call holding time (call duration). As shown in Figure 3.7, call holding time does not demonstrate a strong cyclic pattern.

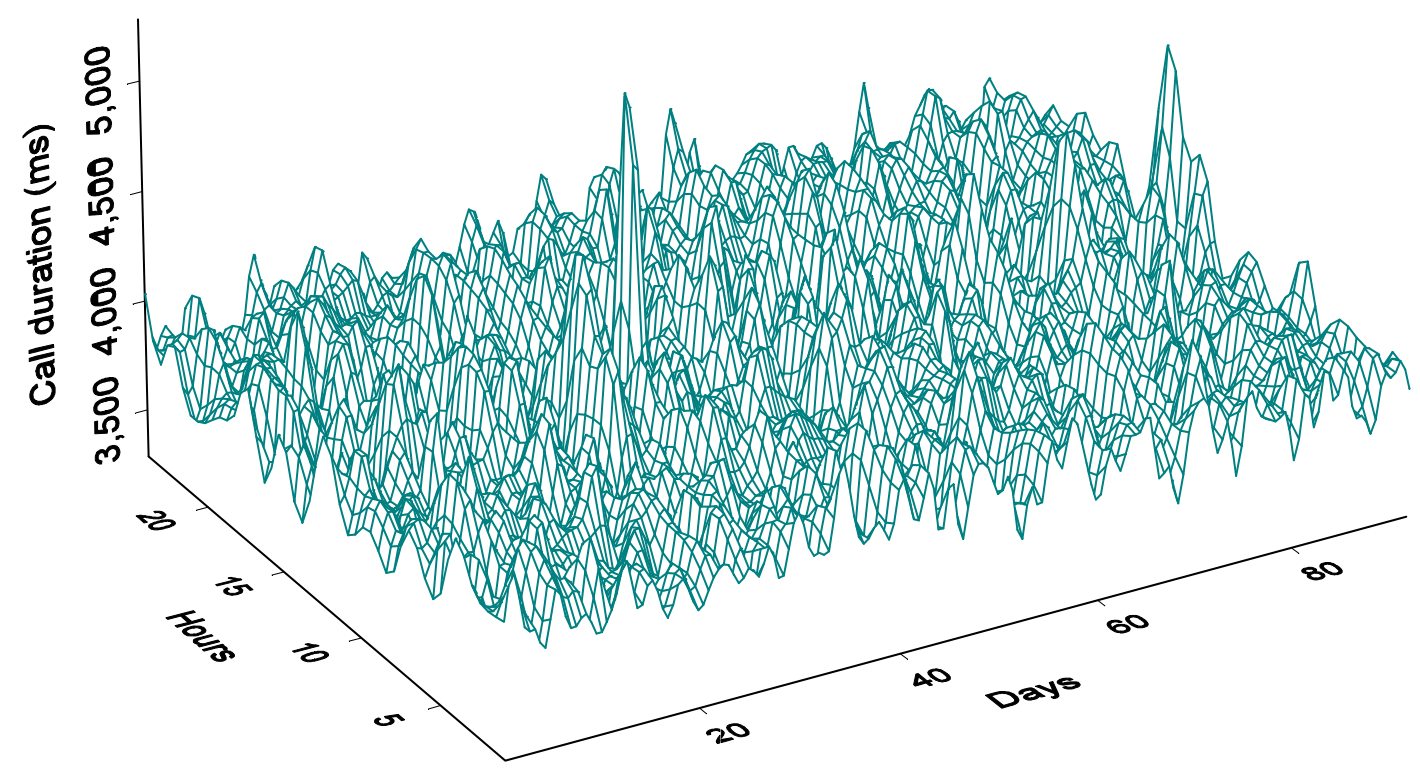

Figure 3.7: Hourly average of call holding time over 92 days in year 2003. 
We use Fourier analysis to analyze the hourly average of call holding time in the frequency domain. Analysis results shown in Figure 3.8 indicate that the average hourly call holding time has a weekly cyclic pattern.

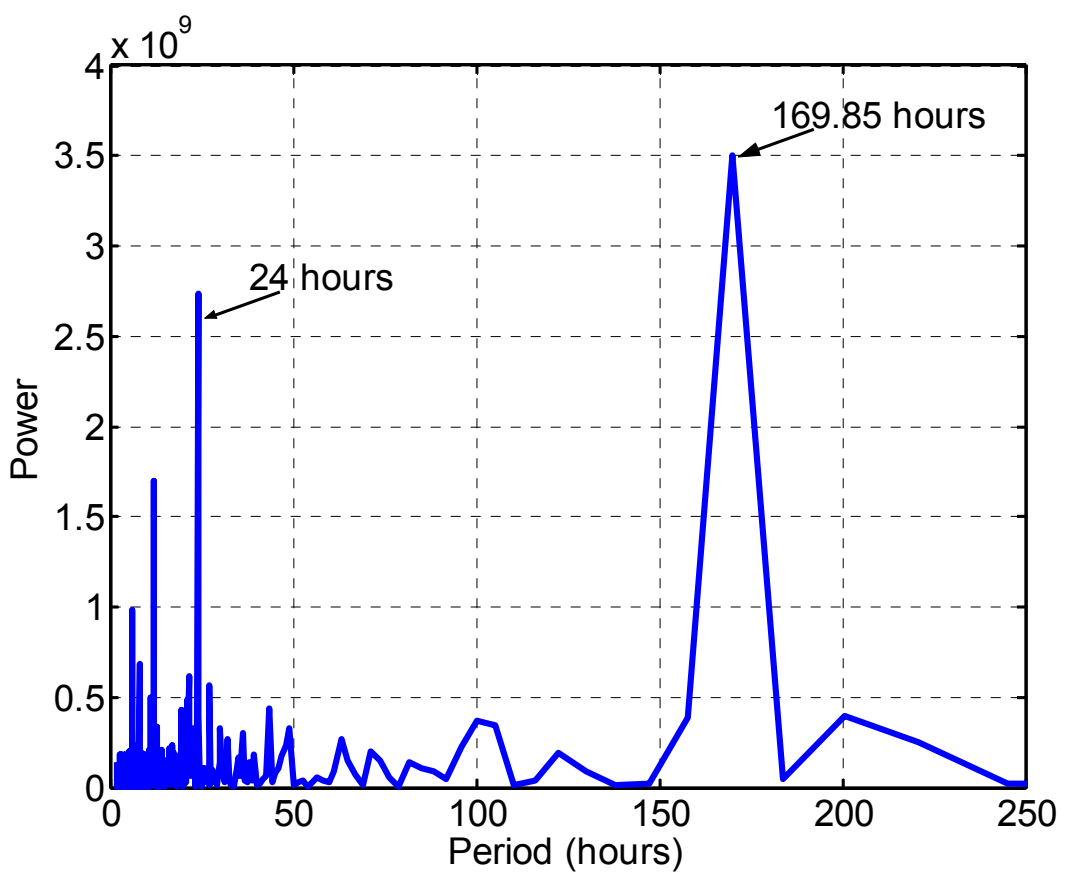

Figure 3.8: Power spectrum of average hourly call holding time indicates a weekly cyclic pattern.

\subsubsection{Agencies and call arrival rates}

The E-Comm network currently serves over ten emergency agencies including Vancouver Police, RCMP, and BC Ambulance. Each emergency agency has a unique agency ID. In this thesis, we use agency IDs to represent emergency agencies. The mapping between agency ID and agency name is not disclosed due to security concerns.

Emergency agencies and their average daily call arrival rate are listed in Table 3.2. We can observe that few heavy user agencies account for most of the call traffic in the E-Comm network. For example, call traffic generated by Agencies 2 and 5 accounts 
for more than $80 \%$ of the overall traffic in both 2002 and 2003 . The call traffic increasing rates vary significantly among user agencies.

Table 3.2: Agencies and average daily call arrival rates.

\begin{tabular}{|c|r|r|r|}
\hline Agency ID & \multicolumn{2}{|c|}{ Average daily call arrival rate } & \multirow{2}{*}{ Change } \\
\cline { 1 - 3 } & Year 2002 & Year 2003 & \\
\hline 1 & 1,360 & 118 & $-91 \%$ \\
\hline 2 & 93 & 307 & $230 \%$ \\
\hline 3 & 27,803 & 27,659 & $-1 \%$ \\
\hline 4 & 1,191 & 1,266 & $6 \%$ \\
\hline 5 & 855 & 894 & $5 \%$ \\
\hline 6 & 25,994 & 48,915 & $88 \%$ \\
\hline 7 & $\mathrm{n} / \mathrm{a}$ & 438 & $\mathrm{n} / \mathrm{a}$ \\
\hline 8 & 38 & 34 & $-11 \%$ \\
\hline 10 & 214 & 1 & $-100 \%$ \\
\hline 11 & 9,439 & 11,692 & $24 \%$ \\
\hline 13 & 1,184 & 656 & $-45 \%$ \\
\hline 14 & 212 & 145 & $-32 \%$ \\
\hline 15 & 78 & 60 & $-23 \%$ \\
\hline 20 & $\mathrm{n} / \mathrm{a}$ & $<1$ & $\mathrm{n} / \mathrm{a}$ \\
\hline 21 & $\mathrm{n} / \mathrm{a}$ & $<1$ & $\mathrm{n} / \mathrm{a}$ \\
\hline Total & $<1$ & 2,999 & $\mathrm{n} / \mathrm{a}$ \\
\hline & 68,462 & 95,184 & $39 \%$ \\
\hline
\end{tabular}

The difference of call arrival rates among user agencies suggests that modeling call traffic on agency level is more accurate than modeling traffic for the entire network. Moreover, heavy user agencies should receive additional attention in traffic modeling.

\subsubsection{Systems and call arrival rates}

The E-Comm network consists of eleven systems. E-Comm system IDs and average daily call arrival rate of each system are listed in Table 3.3. Table 3.3 indicates 
that the overall call traffic increased by $37 \%$ from 2002 to 2003 , which implies that additional channels are required in 2003 in order to maintain the same Grade of Service (GoS). We can also observe that the change of average daily call arrival rate in different systems varies significantly, which implies that each system needs individual assessment when expanding its wireless channels.

Table 3.3: Systems and average daily call arrival rates.

\begin{tabular}{|r|l|r|r|r|}
\hline \multicolumn{2}{|l|}{ System } & \multicolumn{2}{c|}{ Average daily call arrival rate } & \multirow{2}{*}{ Change } \\
\cline { 1 - 2 } ID & Coverage & Year 2002 & Year 2003 & \\
\hline 1 & Vancouver & 53,183 & 56,736 & $7 \%$ \\
\hline 2 & Burnaby & 19,581 & 27,325 & $40 \%$ \\
\hline 3 & Maple Ridge & 7,050 & 8,263 & $17 \%$ \\
\hline 4 & Langley & 7,705 & 18,354 & $138 \%$ \\
\hline 5 & Seymour & 4,241 & 5,325 & $26 \%$ \\
\hline 6 & Port Coquitlam & 15,336 & 22,673 & $48 \%$ \\
\hline 7 & Richmond & 18,752 & 23,846 & $27 \%$ \\
\hline 8 & Mission & 5,929 & 5,706 & $-4 \%$ \\
\hline 9 & Surrey & 12,714 & 31,953 & $151 \%$ \\
\hline 10 & South Surrey & 7,288 & 13,969 & $92 \%$ \\
\hline 11 & Bowen Island & 7,960 & 4,021 & $-49 \%$ \\
\hline & Total & 159,740 & 218,171 & $37 \%$ \\
\hline
\end{tabular}

\subsection{Busy hours}

PSWNs are designed to meet the GoS standards during busy hours. Industry Canada requires that less than $3 \%$ of calls could be queued for an average call holding time during busy hours [7].

The average hourly call arrival rates for the 2002 and 2003 datasets are 2,853 and 3,966 , respectively. Table 3.4 shows that the call arrival rate during busy hours is 
approximately twice the average call arrival rate. For the statistical modeling of call traffic, we only consider busy hour traffic.

Table 3.4: The top three busiest hours in 2002 and 2003 traffic datasets.

\begin{tabular}{|c|c|c|}
\hline Busy hour (BH) & Period & Number of calls \\
\hline $1^{\text {st }} \mathrm{BH}$ in 2002 & 2002-03-01 04:00 - 05:00 & 5506 \\
\hline $2^{\text {nd }} \mathrm{BH}$ in 2002 & $2002-02-28 \quad 22: 00-23: 00$ & 5417 \\
\hline $3^{\text {rd }} \mathrm{BH}$ in 2002 & $2002-03-0122: 00-23: 00$ & 5399 \\
\hline $1^{\text {st }} \mathrm{BH}$ in 2003 & $2003-05-2922: 00-23: 00$ & 8922 \\
\hline $2^{\text {nd }} \mathrm{BH}$ in 2003 & 2003-05-15 02:00 - 03:00 & 8664 \\
\hline $3^{\text {rd }} \mathrm{BH}$ in 2003 & $2003-05-08 \quad 21: 00-22: 00$ & 8471 \\
\hline
\end{tabular}




\section{CHAPTER 4: STATISTICAL TRAFFIC MODELING}

In this Chapter, we first introduce the modeling/simulation approach and describe traditional models for evaluating radio networks. We then describe the process of modeling call holding time and call inter-arrival time. We also address the call coverage issue in the E-Comm network. Two statistical modeling techniques, the KolmogorovSmirnov goodness-of-fit (K-S GoF) test and the Maximum Likelihood Estimation (MLE), are also described.

\subsection{Modeling/simulation approach}

It is important to precisely estimate the maximum network capacity of public safe wireless networks and predict network performance in the future. In other words, it is important for the network operators to know answers for questions such as "How many mobile radios we can put into our network while keeping the busy hour call blocking probability under three percent?" and "How many additional channels are needed when call volume is increased by twenty percent?"

Generally speaking, there are four approaches to study a system and to answer

these questions [8]: (1) experiment with the deployed system, (2) experiment with a physical model of the system (test bed), (3) use an analytical approach to experiment with a mathematical model of the system, and (4) use simulation to experiment with a mathematical model of the system. 
There are three ways to evaluate and predict the E-Comm network performance: utilize existing formulas, employ a modeling/analytical approach, and/or use a modeling/simulation approach. E-Comm operators currently use existing formulas such as Erlang B and Erlang $\mathrm{C}$ to evaluate their network performance. However, we will show in Section 4.2 that this approach has several limitations and cannot provide reliable estimation results.

One way to predict future network traffic is to study the past traffic and extract past patterns and relationships. These patterns and relationships form a model. The modeling process provides a means of creating simplified representations of the "real network". Once the mathematical model of the target network is built, we may either use an analytical approach or a simulation approach to evaluate and predict the network performance. In this thesis, we adopt the modeling/simulation approach because of its capability to handle complex mathematical models.

To build the E-Comm traffic model, we first study the traffic data collected from the E-Comm network. We then select several candidate distributions, estimate their parameters, identify the most suitable distribution, and build a statistical model that represents the usage of the E-Comm network.

\subsection{Introduction to traditional traffic models}

\subsubsection{Erlang models}

Erlang B and Erlang C models are widely used by call traffic engineers to plan the network resources, such as number of phone lines in Public Switched Telephone Network (PSTN), Private Branch Exchange (PBX) capacity of call centers, and number of radio 
channels in cellular networks [10]. Erlang models are named after the Danish mathematician A. K. Erlang.

Erlang is used as a dimensionless unit in traffic engineering to measure traffic intensity. It measures continuous use of one circuit. For example, call traffic generated by a one hour long call is one Erlang. Call traffic generated by two 30 minutes long calls is also one Erlang.

Mathematical representations of Erlang $\mathrm{B}$ and Erlang $\mathrm{C}$ models are given by Equations (4-1) and (4-2), respectively. In both equations, $N$ is the total number of resources in the system, such as channels or lines, and $A$ is the total traffic volume measured in Erlang.

In Erlang B model:

$$
P_{B}=\frac{\frac{A^{N}}{N !}}{\sum_{x=0}^{N} \frac{A^{x}}{x !}}
$$

$P_{B}$ is the probability that a call request will be rejected due to lack of resources.

In Erlang C model:

$$
P_{C}=\frac{\frac{A^{N}}{N !} \frac{N}{N-A}}{\sum_{x=0}^{N-1} \frac{A^{x}}{x !}+\frac{A^{N}}{N !} \frac{N}{N-A}},
$$

$P c$ is the probability that a call request will be delayed in obtaining a resource.

Erlang B and Erlang C models assume (1) infinite number of call sources or that the number of call sources is much larger than the number of available channels, (2) 
Poisson distributed call arrival, and (3) exponentially distributed call holding time. The Erlang B model further assumes that (4) a blocked call will be rejected immediately and (5) a user will not retry the same request after being rejected. The Erlang C model further assumes that (6) a blocked call will be put into a FIFO queue with infinite size and that (7) a user will not abandon the call request until the call is successfully completed.

\subsubsection{Failure of Erlang models}

In the E-Comm network, although assumptions (1) and (2) are likely to be valid, assumptions (3) through (7) are not applicable. Moreover, Erlang models were originally developed for traditional networks where there is no group call, which implies that caller and callee have a one-to-one relationship. However, calls in the E-Comm network are all group calls (one-to-many caller-to-callee relationship) and most calls use more than one channel simultaneously. These indicate that Erlang models are not suitable for the EComm network.

\subsection{Traffic modeling on agency level}

We showed previously in Table 3.2 that the majority number of calls is initiated by few user agencies. We further investigate the number of calls made by each agency during the top three busiest hours in 2002 and 2003 datasets. 
Table 4.1: Aggregated number of calls during the top three busiest hours in 2002 and 2003.

\begin{tabular}{|c|c|c|c|c|}
\hline Agency ID & Year 2002 & Percentage & Year 2003 & Percentage \\
\hline $\mathrm{n} / \mathrm{a}$ & 72 & $0.4 \%$ & 17 & $0.1 \%$ \\
\hline 1 & 5 & $0.0 \%$ & 19 & $0.1 \%$ \\
\hline 2 & 7,222 & $44.2 \%$ & 7,976 & $30.6 \%$ \\
\hline 3 & 363 & $2.2 \%$ & 464 & $1.8 \%$ \\
\hline 4 & 60 & $0.4 \%$ & 180 & $0.7 \%$ \\
\hline 5 & 6,289 & $38.5 \%$ & 14,183 & $54.4 \%$ \\
\hline 6 & 0 & $0.0 \%$ & 119 & $0.5 \%$ \\
\hline 7 & 4 & $0.0 \%$ & 0 & $0.0 \%$ \\
\hline 8 & 22 & $0.1 \%$ & 0 & $0.0 \%$ \\
\hline 10 & 1,878 & $11.5 \%$ & 2,278 & $8.7 \%$ \\
\hline 11 & 308 & $1.9 \%$ & 161 & $0.6 \%$ \\
\hline 13 & 28 & $0.2 \%$ & 25 & $0.1 \%$ \\
\hline 14 & 71 & $0.4 \%$ & 37 & $0.1 \%$ \\
\hline 15 & 0 & $0.0 \%$ & 0 & $0.0 \%$ \\
\hline 20 & 0 & $0.0 \%$ & 0 & $0.0 \%$ \\
\hline 21 & 0 & $0.0 \%$ & 598 & $2.3 \%$ \\
\hline Total & 16,322 & $100 \%$ & 26,057 & $100 \%$ \\
\hline
\end{tabular}

Table 4.1 indicates that during busy hours, more than $80 \%$ of the calls are initiated by Agencies 2 and 5. Since these two heavy user agencies have larger impact on the network than the remaining of the agencies, to simplify the traffic modeling process, we regroup the agencies into three new agencies and model their call traffic. Table 4.2 shows the mapping between the original and the new agency IDs.

Table 4.2: Regrouped agencies and the original agencies.

\begin{tabular}{|c|c|}
\hline Virtual agency ID & Original agency ID \\
\hline A & 2 \\
\hline B & 5 \\
\hline C & (all other agencies) \\
\hline
\end{tabular}




\subsection{Modeling call holding time}

Before we start describing the modeling of call holding time, we introduce two related statistical modeling techniques: Kolmogorov-Smirnov goodness-of-fit (K-S GoF) test and Maximum Likelihood Estimation (MLE).

\subsubsection{Maximum likelihood estimation}

Introduced by R. A. Fisher in 1920s, MLE [9] is currently the most popular method of estimating distribution parameters. The goal of MLE is to find the parameters that make the candidate distribution fit the observed data best.

Let $f_{\theta}(x)=f(x \mid \theta)$ be the probability distribution function, where $\theta=\left(\theta_{1}, \theta_{2}, \ldots, \theta_{m}\right)$ is the parameter vector. Likelihood of $\theta$ is defined as

$$
\operatorname{like}(\theta)=\prod_{i=1}^{n} f\left(x_{i} \mid \theta\right) \text {. }
$$

Since the maximum product is difficult to calculate and logarithm is an increasing function, we use the log-likelihood $l(\theta)$ instead. The parameter $\theta$ that maximizes $l(\theta)$ will also maximize like $(\theta)$ :

$$
\begin{aligned}
l(\theta) & =\log \left(\prod_{i=1}^{n} f\left(x_{i} \mid \theta\right)\right) \\
& =\sum_{i=1}^{n} \log \left(f\left(x_{i} \mid \theta\right)\right)
\end{aligned}
$$

We use the Poisson distribution as an example to demonstrate how MLE works. The Probability Density Function (PDF) for the Poisson distribution is

$$
p(x)=\frac{\lambda^{x} e^{-\lambda}}{x !}
$$


The log-likelihood can be deduced as

$$
\begin{aligned}
l(\lambda) & =\sum_{i=1}^{n}\left(x_{i} \log \lambda-\lambda-\log x_{i} !\right) \\
& =\log \lambda \sum_{i=1}^{n} x_{i}-n \lambda-\sum_{i=1}^{n} \log x_{i} !
\end{aligned}
$$

To find the maximum of $l(\theta)$, we final its derivative and set it to zero:

$$
\begin{aligned}
\frac{\partial l(\lambda)}{\partial \lambda} & =\frac{1}{\lambda} \sum_{i=1}^{n} x_{i}-n . \\
& =0
\end{aligned}
$$

Equation (4-6) implies $\hat{\lambda}=\bar{X}$. However, in most other cases, $l(\theta)$ is not deducible mathematically. We use a software tool called mle [12] to estimate the parameters.

\subsubsection{Kolmogorov-Smirnov goodness-of-fit test}

Goodness-of-fit test (GoF) is used to statistically verify whether or not a sample dataset $X_{1}, X_{2}, \ldots, X_{n}$ follows a particular distribution with distribution function $F$. In other words, the GoF test is used to test the following null hypothesis $H_{0}$ :

$\mathrm{H}_{0}: X_{1}, X_{2}, \ldots, X_{n}$ are independent random variables with a specific distribution $F(x)$

The Chi-square test [13] and the Kolmogorov-Smirnov test [13] are the most popular goodness-of-fit tests. The Chi-square test is suitable for testing discrete random variables, while the K-S test is more suitable for testing continuous random variables. Since our objects (call holding time and call inter-arrival time) are both modeled using continuous random variables, we use the K-S test. 
The K-S GoF test is based on the empirical distribution function $F_{e}$. Suppose that we have $n$ independent random variables $X_{1}, X_{2}, \ldots, X_{n}$. Since the order of these variables does not affect the underlying distribution function, we further assume that $X_{1}, X_{2}, \ldots, X_{n}$ are in an increasing order. The Empirical Distribution Function (ECDF) $F_{e}$ is defined as:

$$
F_{e}(x)=\frac{\text { total number of } i \text { where } Y_{i} \leq x}{n} .
$$

The Kolmogorov-Smirnov test statistic D is defined as:

$$
\begin{aligned}
D & =\underset{x}{\operatorname{Supremum}}\left|F_{e}(x)-F(x)\right| \\
& =\operatorname{Supremum}\left\{\operatorname{Supremum}\left\{F_{e}(x)-F(x)\right\}, \text { Supremum }\left\{F(x)-F_{e}(x)\right\}\right\} \\
& =\operatorname{Supremum}\left\{\frac{j}{n}-F\left(Y_{j}\right), F\left(Y_{j}\right)-\frac{(j-1)}{n}, j=1, \ldots, n\right\} .
\end{aligned}
$$

Equation (4-8) states that a large value of $D$ indicates a poor fit of $F_{e}(x)$ and $F(x) \cdot p$-value,

defined as $p$-value $=P_{F}\{D \geq d\}[11]$, is an indicator of how likely $F(x)$ is accepted. Null hypothesis $\mathrm{H}_{0}$ is likely to be accepted when $p$-value $>0.01$ and $\mathrm{H}_{0}$ is very likely to be accepted when $p$-value $>0.05$.

\subsubsection{Survey of existing models for call holding time}

Over the years, a number of probability models have been developed for call holding time in various networks such as PSTN and cellular networks. Bolotin shows [14] that the exponential distribution underestimates the proportion of short calls in traditional PSTNs. Bolotin further shows [15] that the call holding time in traditional PSTNs follows lognormal distribution with a mean value of 200-300 seconds. In [16]- 
[19], Jordán and Barceló show that Erlang-jk and mixture of lognormals fit very well voice holding time in Public Access Mobile Radio (PAMR) systems with mean value of 20-40 seconds. Using field data from cellular telephony systems, Jedrzycki and Leung find that the lognormal distribution provides a better fit for the channel holding time [20]. In [21], [22], Orlik and Rappaport proposed a model called the Sum of the HyperExponential (SOHYP) to model the channel holding time in data used in [20]. Fang et al., proposed the Hyper-Erlang distribution [23]-[25] to model cell residence time that may be used to better characterize channel holding time in mobile computing and Personal Communication Services (PCS) networks.

Traffic data from PSWNs are rarely available due to their confidential nature and, hence, few models of call holding time have been developed for such networks. The large difference between mean call holding times in PSWNs and call holding times in PSTN/Cellular networks suggests that call holding time models developed for other networks might not be suitable for PSWNs. A recent study of E-Comm's aggregated traffic shows that the call holding time during busy hours fits the lognormal distribution [27]. However, to the author's knowledge, there is no published work dealing with modeling call traffic in PSWNs on user agency level.

\subsubsection{Model comparison and selection}

In this Section, we investigate the call holding time of E-Comm traffic on the user agency level. The candidate distributions to be tested and their PDFs are listed in Table 4.3. 
Table 4.3: Candidate distributions for call holding time.

\begin{tabular}{|l|l|}
\hline Distribution & Probability density function \\
\hline Exponential & $f(x)=\frac{1}{\beta} e^{-\frac{x}{\beta}}$ \\
\hline Lognormal & $f(x)=\frac{1}{x \sqrt{2 \pi \sigma^{2}}} e^{\frac{(\ln (x)-\mu)^{2}}{-2 \sigma^{2}}}$ \\
\hline Gamma & $f(x)=\beta^{-k} \frac{x^{k-1}}{(k-1) !} e^{-\frac{x}{\beta}}$ \\
\hline
\end{tabular}

To fit and compare candidate distributions with E-Comm traffic data, we first extract 500 sequential data samples (call holding times) from the traffic data table. We then select a candidate distribution and use MLE to estimate its parameters. The K-S GoF test is then used to evaluate the candidate distribution. When $p$-value is greater than 0.03 , the candidate distribution is likely to be accepted. Otherwise, the candidate distribution is likely to be rejected.

We fit candidate distributions with call holding times of Agencies A, B, and C during the top three busiest hours in the 2002 dataset. Estimated distribution parameters and K-S test results are listed in Table 4.4. Cells with $p$-value $>0.03$ are marked in grey. $\mathrm{K}-\mathrm{S}$ test results ( $p$-values) shown in Table 4.4 indicate that the lognormal distribution is ideal to model the call holding time for Agency A. 
Table 4.4: Test of candidate distributions for call holding time of Agency A during busy hours in the 2002 dataset.

\begin{tabular}{|l|l|l|l|}
\hline \multirow{2}{*}{ Distributions } & \multicolumn{3}{|l|}{ Distribution parameters and K-S test results } \\
\cline { 2 - 4 } & Busy hour 1 & Busy hour 2 & Busy hour 3 \\
\hline Exponential & $\beta=3683.6$ & $\beta=3664.06$ & $\beta=3658.56$ \\
& $p$-value $=0$ & $p$-value $=0$ & $p$-value =0 \\
\hline Lognormal & $\sigma=8.0538$ & $\sigma=8.0378$ & $\sigma=7.9872$ \\
& $\mu=0.5505$ & $\mu=0.5769$ & $\mu=0.6334$ \\
& $p$-value $=0.0832$ & $p$-value $=0.4952$ & $p$-value =0.2634 \\
\hline Gamma & $\beta=1108.0657$ & $\beta=1173.6163$ & $\beta=1493.315$ \\
& $k=3.3246$ & $k=3.1222$ & $k=2.4501$ \\
& $p$-value $=0.0002$ & $p$-value $=0.0137$ & $p$-value =0.0027 \\
\hline
\end{tabular}

K-S test results shown in Table 4.5 indicate that although the lognormal distribution does not fit the call holding time of Agency B at 3\% significance level all the time, it is nevertheless adequate to model it call holding time.

Table 4.5: Test of candidate distributions for call holding time of Agency B during the top three busy hours in the 2002 dataset.

\begin{tabular}{|l|l|l|l|}
\hline \multirow{2}{*}{ Distributions } & \multicolumn{3}{|l|}{ Distribution parameters and K-S test results } \\
\cline { 2 - 4 } & Busy hour 1 & Busy hour 2 & Busy hour 3 \\
\hline Exponential & $\beta=4269.78$ & $\beta=4198.82$ & $\beta=3742.3$ \\
& $p$-value $=0$ & $p$-value $=0$ & $p$-value $=0$ \\
\hline Lognormal & $\sigma=8.0882$ & $\sigma=8.0856$ & $\sigma=7.9663$ \\
& $\mu=0.7256$ & $\mu=0.7232$ & $\mu=0.7312$ \\
& $p$-value $=0.0258$ & $p$-value $=0.4522$ & $p$-value $=0.0474$ \\
\hline Gamma & $\beta=2141.4626$ & $\beta=2002.4384$ & $\beta=1811.9239$ \\
& $k=1.994$ & $k=2.097$ & $k=2.0655$ \\
& $p$-value $=0$ & $p$-value $=0.0012$ & $p$-value $=0.0001$ \\
\hline
\end{tabular}

K-S test results shown in Table 4.6 indicate that no candidate distribution fits the call holding time of Agency $\mathrm{C}$ at satisfying significance levels. However, the lognormal distribution still performs better than other candidate distributions. 
Table 4.6: Test of candidate distributions for call holding time of Agency $C$ during the top three busy hours in the 2002 dataset.

\begin{tabular}{|l|l|l|l|}
\hline \multirow{2}{*}{ Distributions } & \multicolumn{3}{|l|}{ Distribution parameters and K-S test results } \\
\cline { 2 - 4 } & Busy hour 1 & Busy hour 2 & Busy hour 3 \\
\hline Exponential & $\beta=3505.58$ & $\beta=4014.54$ & $\beta=3786.8$ \\
& $p$-value $=0$ & $p$-value $=0$ & $p$-value $=0$ \\
\hline Lognormal & $\sigma=7.8759$ & $\sigma=8.094$ & $\sigma=7.9621$ \\
& $\mu=0.8154$ & $\mu=0.6228$ & $\mu=0.7251$ \\
& $p$-value $=0$ & $p$-value $=0.0036$ & $p$-value =0.0002 \\
\hline Gamma & $\beta=1848.5619$ & $\beta=1539.0157$ & $\beta=1938.2806$ \\
& $k=1.8965$ & $k=2.6087$ & $k=1.9538$ \\
& $p$-value $=0$ & $p$-value $=0$ & $p$-value =0 \\
\hline
\end{tabular}

Because the two heavy user agencies (Agencies A and B) account for $80-90 \%$ of the total call traffic and their call holding time follows lognormal distribution, we adopt the lognormal distribution to model call holding time in simulations.

\subsection{Modeling call inter-arrival time}

\subsubsection{Survey of existing models for call inter-arrival time}

The Poisson model for call arrival time is widely used in PSTNs [28]. It implies that the inter-arrival time is exponentially distributed. Exponentially distributed call interarrival time was originally assumed in cellular networks. It was shown [29]-[31] that exponential distribution is only suitable for modeling inter-arrival times of "fresh" (new) calls in micro-cellular networks. However, exponential distribution is not suitable for modeling all (new and hand-over) call inter-arrivals in micro-cellular networks due to the frequent call handovers. 
The E-Comm network is a macro-cellular network where call handover rarely happens. Call inter-arrival time in the E-Comm network was found to be exponentially distributed [27].

\subsubsection{Model comparison and selection}

In this Section, we investigate the call inter-arrival time of E-Comm traffic on the user agency level. Candidate distributions are listed in Table 4.7.

Table 4.7: Candidate distributions for call inter-arrival time.

\begin{tabular}{|l|l|}
\hline Distribution & Probability density function \\
\hline Exponential & $f(x)=\frac{1}{\beta} e^{-\frac{x}{\beta}}$ \\
\hline Lognormal & $f(x)=\frac{1}{x \sqrt{2 \pi \sigma^{2}}} e^{\frac{(\ln (x)-\mu)^{2}}{-2 \sigma^{2}}}$ \\
\hline Gamma & $f(x)=\beta^{-k} \frac{x^{k-1}}{(k-1) !} e^{-\frac{x}{\beta}}$ \\
\hline
\end{tabular}

The method we employ to evaluate candidate distributions of call inter-arrival time is similar to the method used to evaluate call holding time. Estimated distribution parameters and K-S test results are listed in Tables 4.8-4.10. Cells with $p$-value $>0.03$ are marked in grey.

The results shown in Tables 4.8 and 4.9 indicate that both exponential distribution and gamma distribution are adequate to model the call inter-arrival time of Agencies A and $\mathrm{B}$. This is expected since the gamma distribution is a more general form of exponential distribution. We use the exponentially distributed call inter-arrival time in later simulations because of its simplicity. 
Table 4.8: Test of candidate distributions for call inter-arrival time of Agency A during the top three busy hours in the 2002 dataset.

\begin{tabular}{|l|l|l|l|}
\hline \multirow{2}{*}{ Distributions } & \multicolumn{3}{|l|}{ Distribution parameters and K-S test results } \\
\cline { 2 - 4 } & Busy hour 1 & Busy hour 2 & Busy hour 3 \\
\hline Exponential & $\beta=1138.674$ & $\beta=2010.986$ & $\beta=1282.2244$ \\
& $p$-value $=0.0019$ & $p$-value $=0.7604$ & $p$-value $=0.1232$ \\
\hline Lognormal & $\sigma=6.6043$ & $\sigma=7.0211$ & $\sigma=6.6795$ \\
& $\mu=1.0791$ & $\mu=1.2888$ & $\mu=1.1643$ \\
& $p$-value $=0.0001$ & $p$-value $=0.0002$ & $p$-value =0.0009 \\
\hline Gamma & $\beta=879.3113$ & $\beta=2036.5336$ & $\beta=1079.9108$ \\
& $k=1.2951$ & $k=0.9875$ & $k=1.1874$ \\
& $p$-value $=0.4591$ & $p$-value $=0.6606$ & $p$-value =0.7863 \\
\hline
\end{tabular}

Table 4.9: Test of candidate distributions for call inter-arrival time of Agency B during the top three busy hours in the 2002 dataset.

\begin{tabular}{|l|l|l|l|}
\hline \multirow{2}{*}{ Distributions } & \multicolumn{3}{|l|}{ Distribution parameters and K-S test results } \\
\cline { 2 - 4 } & Busy hour 1 & Busy hour 2 & Busy hour 3 \\
\hline Exponential & $\beta=2016.806$ & $\beta=1437.12$ & $\beta=1738.2826$ \\
& $p$-value $=0.093$ & $p$-value $=0.992$ & $p$-value =0.5093 \\
\hline Lognormal & $\sigma=7.1558$ & $\sigma=6.72$ & $\sigma=6.8838$ \\
& $\mu=1.0865$ & $\mu=1.2406$ & $\mu=1.2564$ \\
& $p$-value $=0.0017$ & $p$-value $=0.0103$ & $p$-value =0.0007 \\
\hline Gamma & $\beta=1623.0727$ & $\beta=1377.4624$ & $\beta=1737.6351$ \\
& $k=1.2427$ & $k=1.0434$ & $k=1.0004$ \\
& $p$-value $=0.8678$ & $p$-value $=0.999$ & $p$-value =0.509 \\
\hline
\end{tabular}

Results shown in Table 4.10 indicate that although Gamma distribution may occasionally model the distribution of call inter-arrival time of the light user Agency C, no candidate distribution is likely to be accepted. We assume exponentially distributed call inter-arrival time for Agency $\mathrm{C}$ in simulations because Agency $\mathrm{C}$ accounts for only approximately $10 \%$ of the call traffic and has much less impact on E-Comm network than Agencies A and B. 
Table 4.10: Test of candidate distributions for call inter-arrival time of Agency $\mathrm{C}$ during the top three busy hours in the 2002 dataset.

\begin{tabular}{|l|l|l|l|}
\hline \multirow{2}{*}{ Distributions } & \multicolumn{3}{|l|}{ Distribution parameters and K-S test results } \\
\cline { 2 - 4 } & Busy hour 1 & Busy hour 2 & Busy hour 3 \\
\hline Exponential & $\beta=4941.8036$ & $\beta=3303.4309$ & $\beta=4897.246$ \\
& $p$-value $=0$ & $p$-value $=0$ & $p$-value $=0$ \\
\hline Lognormal & $\sigma=7.7809$ & $\sigma=7.5571$ & $\sigma=7.8174$ \\
& $\mu=1.2987$ & $\mu=1.2046$ & $\mu=1.3028$ \\
& $p$-value $=0.0001$ & $p$-value $=0.0002$ & $p$-value =0.0001 \\
\hline Gamma & $\beta=6050.6496$ & $\beta=3140.9854$ & $\beta=5661.0249$ \\
& $k=0.8168$ & $k=1.0518$ & $k=0.8651$ \\
& $p$-value $=0$ & $p$-value $=0.337$ & $p$-value $=0.0064$ \\
\hline
\end{tabular}

\subsection{Call coverage pattern}

The call coverage pattern is important in simulating the E-Comm network because all calls in the E-Comm network are group calls and most of them cover more than one system. As previously discussed in Section 2.1.6, we might underestimate the call blocking probability if we ignore the group calls and investigate only one system.

The call coverage pattern in the E-Comm network is largely determined by the organizations of talk groups and the radio device deployment of user agencies. Factors that affect the call coverage pattern are unknown. In simulations of the E-Comm network, we assume that the pattern of call coverage and the corresponding probability of occurrences remain unchanged. A sample of call coverage pattern is shown in Table 4.11.

Table 4.11: A sample of call coverage pattern of Agency A during the top three busy hours in 2003.

\begin{tabular}{|l|r|r|}
\hline \multicolumn{1}{|c|}{ Call coverage } & No. of occurrences & Percentage \\
\hline System No. 1, 2, 3 & 45 & $1.35 \%$ \\
\hline System No. 1, 3, 8, 10,11 & 69 & $2.07 \%$ \\
\hline System No. 3, 5, 7, 9, 10 & 236 & $7.08 \%$ \\
\hline$\ldots$ & $\ldots$ & $\ldots$ \\
\hline
\end{tabular}




\section{CHAPTER 5: WARNSIM: A SIMULATOR FOR PSWN}

In this Chapter, we introduce the design, implementation, and validation of WarnSim.

\subsection{Overview}

Several network simulation tools are currently available for simulating packet switched networks: OPNET [32], ns-2 [33], and J-SIM [34]. However, to the author's knowledge, there is no simulation software tool publicly available for simulating circuit switched networks such as PSWNs. Moreover, it is inefficient to use a simulator designed for packet switched networks to mimic and simulate circuit switched networks. Hence, we developed WarnSim, an effective, flexible, and easy to use simulation tool to simulate PSWN systems.

WarnSim is a publicly available tool designed to simulate circuit switched radio networks [35], [36]. It can simulate various network sizes (number of cells in a network) and cell capacities (number of channels in cells). Traffic traces are easily imported or can be modeled with a variety of statistical distributions. These features make WarnSim preferable for simulating wide area radio networks, such as PSWNs.

WarnSim is developed using Microsoft Visual C\# .NET, which has a comparable performance to Java [37]. WarnSim works on Windows platforms with .NET framework support and comes with a graphical user interface. 


\subsection{Modules and call flowchart}

WarnSim consists of seven modules, shown in Figure 5.1.

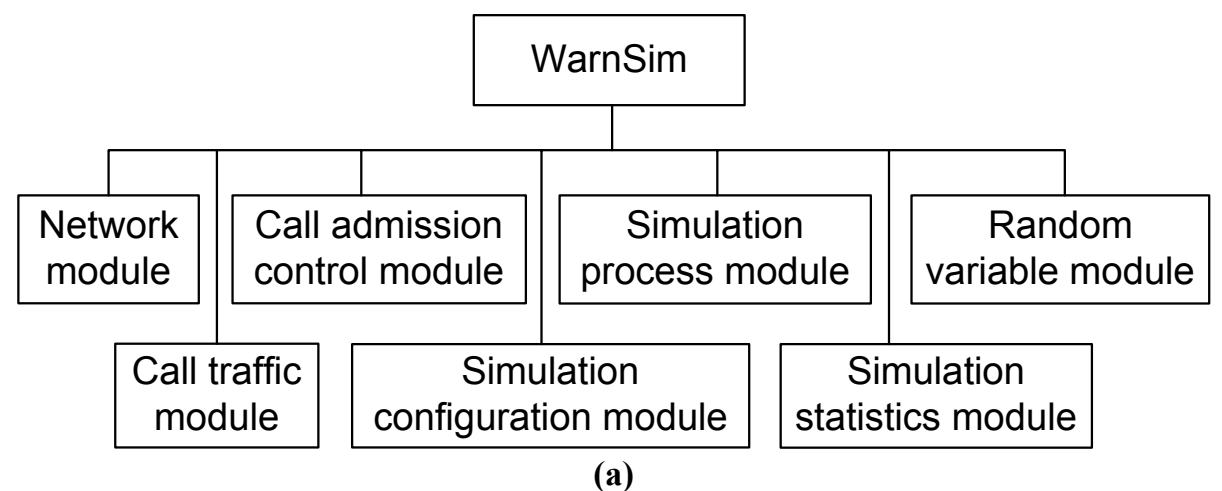

(a)

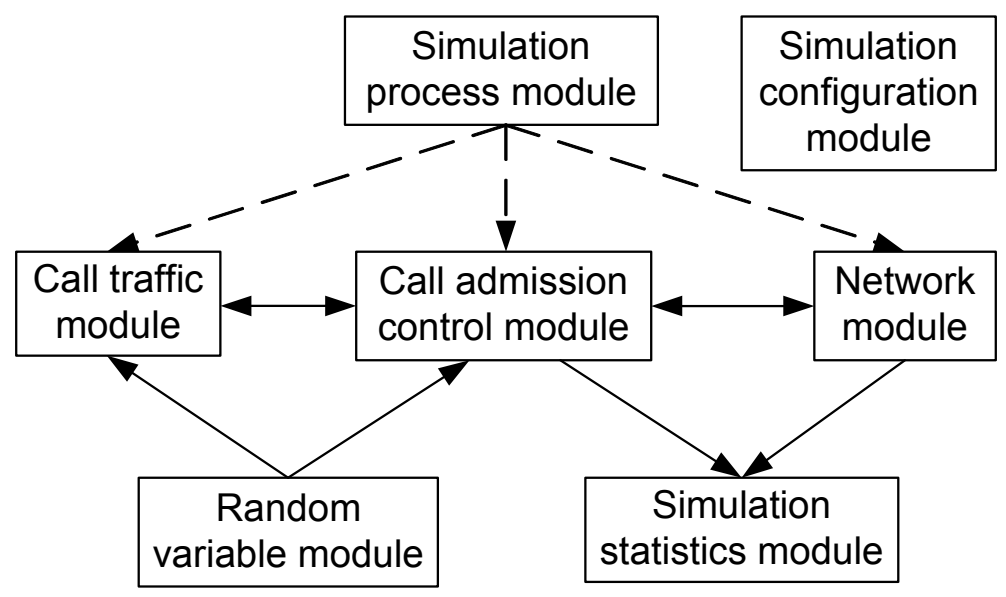

(b)

Figure 5.1: WarnSim: (a) module hierarchical diagram and (b) relationship diagram.

Network module models the cells of a PSWN. A network module may consist of several cells. Each cell has one or more channels with the attribute "busy duration". A channel is free if its "busy duration" is zero. Otherwise, the channel is occupied for the duration of time specified by the "busy duration". The network module distributes calls to cells, tracks the status of the system (number of free or occupied channels), and periodically updates the channel status in every cell. 
Call traffic module prepares call traffic for simulation. It can import traffic trace files from text files/databases or generate call traffic based on user-defined distributions and combine them during a single simulation run.

Call admission control module uses the calls from the call traffic module and checks with the network module to determine if there are available channels for a call to be established. It also manages the retrying mechanism of blocked calls.

Simulation configuration module keeps track of call queuing mechanism and parameters, such as the maximum call queuing time, simulation duration, and simulation granularity.

Simulation process module uses a global timer to control and synchronize the operation of the WarnSim modules.

Simulation statistics module collects real-time and summary statistics (number of calls, blocked calls, call blocking probability, channel utilization, cumulative number of calls, cumulative blocked calls, cumulative call blocking probability, and cumulative channel utilization) from other modules. It is also used to display and visualize simulation results.

Random variable module generates random numbers and random variables. We adopted the MT random number generator [38], which is suitable for stochastic simulations. We also implemented seven types of random variables: uniform, exponential, gamma, normal, lognormal, loglogistic, and Weibull. 
A high level diagram illustrating the call flow mechanism of WarnSim is given in Figure 5.2. The basic functionality of WarnSim is described by the pseudo-code, as shown in Figure 5.3. 


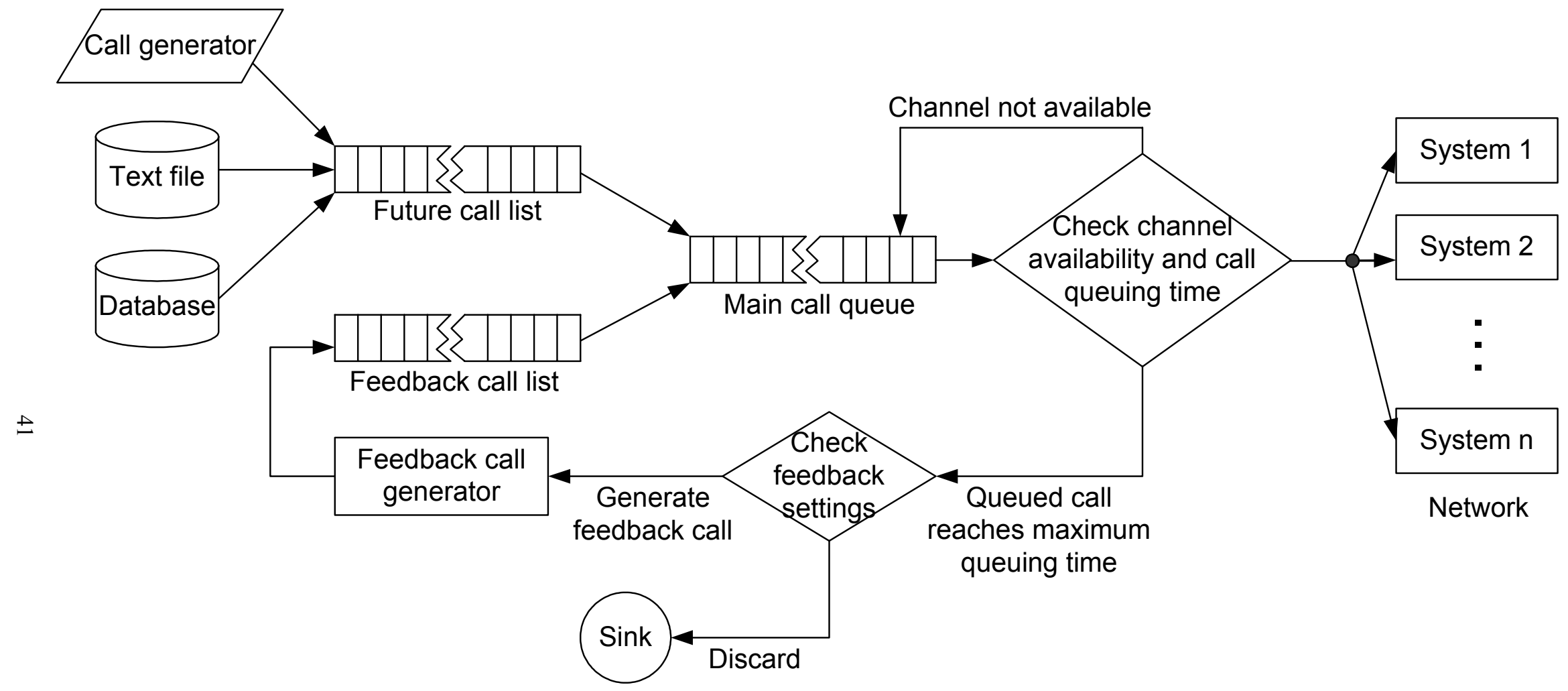

Figure 5.2: High level diagram of the WarnSim simulator. 


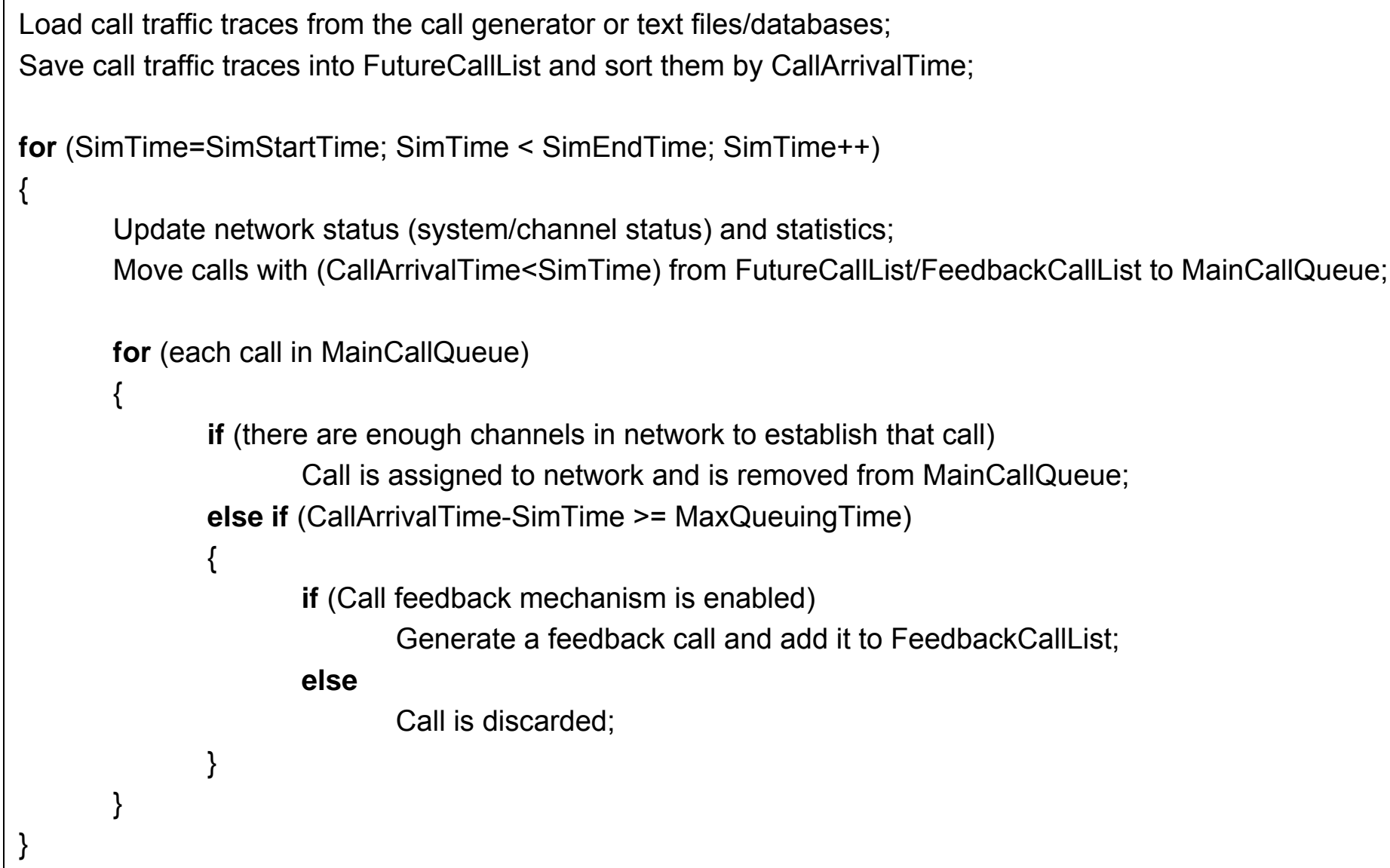

Figure 5.3: Pseudo-code corresponding to the high level diagram of the WarnSim simulator. 


\subsection{WarnSim simulation steps}

Most simulations with WarnSim include five main steps:

Step 1: Network topology setup

add/remove/configure systems

load/save network topology

Step 2: Traffic sources setup

add/remove/configure traffic sources from a text file or a database

add/remove/configure call traffic generators

load/save traffic sources

Step 3: Simulation parameters configuration

configure the length (time span) of simulation

configure calls queuing mechanism

configure dropped calls retrying mechanism

configure statistics collection interval

Step 4: Run simulation

run/stop simulation

pause simulation to view intermediate simulation results

Step 5: Analyze simulation results

list/save simulation results 
plot/export simulation results.

\subsection{WarnSim interface}

WarnSim includes a graphical user interface. Figures 5.4-5.8 show four WarnSim screens during a simulation experiment. Systems in WarnSim are configured in a star network topology, as shown in Figure 5.4 (a). Figure 5.4 (b) shows the WarnSim network configuration screen capturing network information (cells and number of channels). A popup window used to configure the system ID, system name, and number of channels is also shown in Figure 5.4 (b). Figures 5.5 and 5.6 show the configuration screens for traffic sources. A list of traffic sources and popup windows used to import/generate/configure traffic traces are also shown. Figures 5.7 (a) and (b) capture the simulation results screens for channel utilization vs. simulation time. Figures 5.8 (a) and (b) show the simulation results for discarded calls vs. simulation time. 


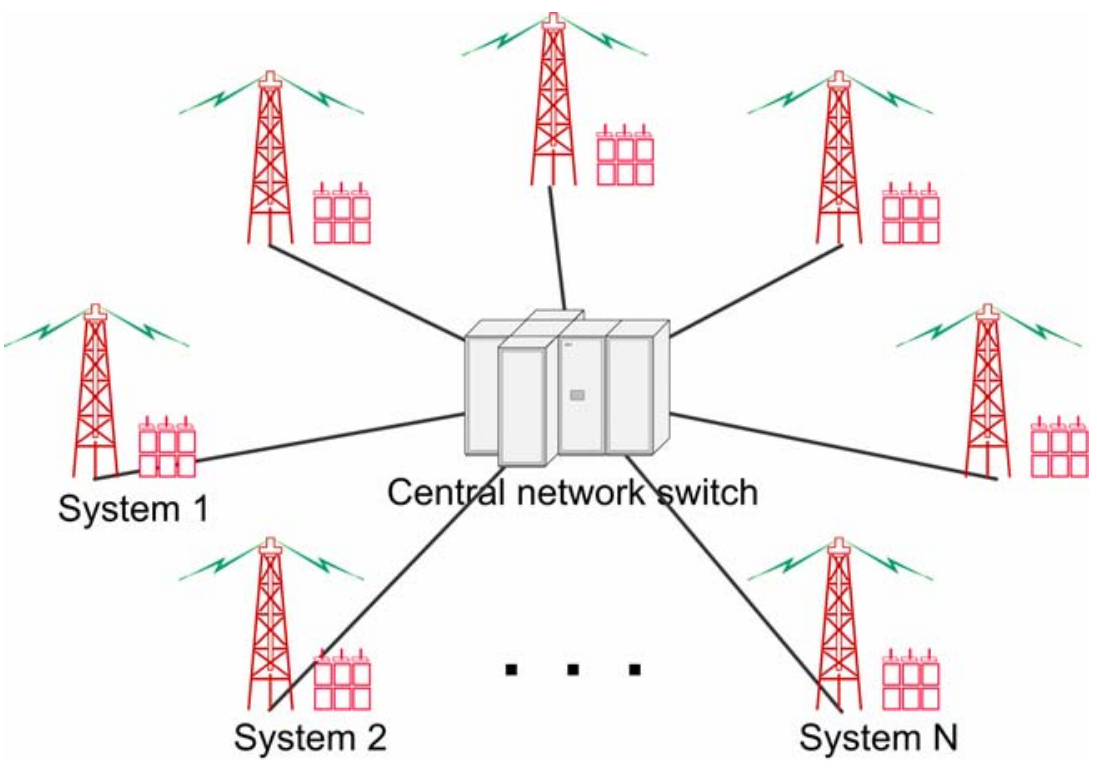

(a)

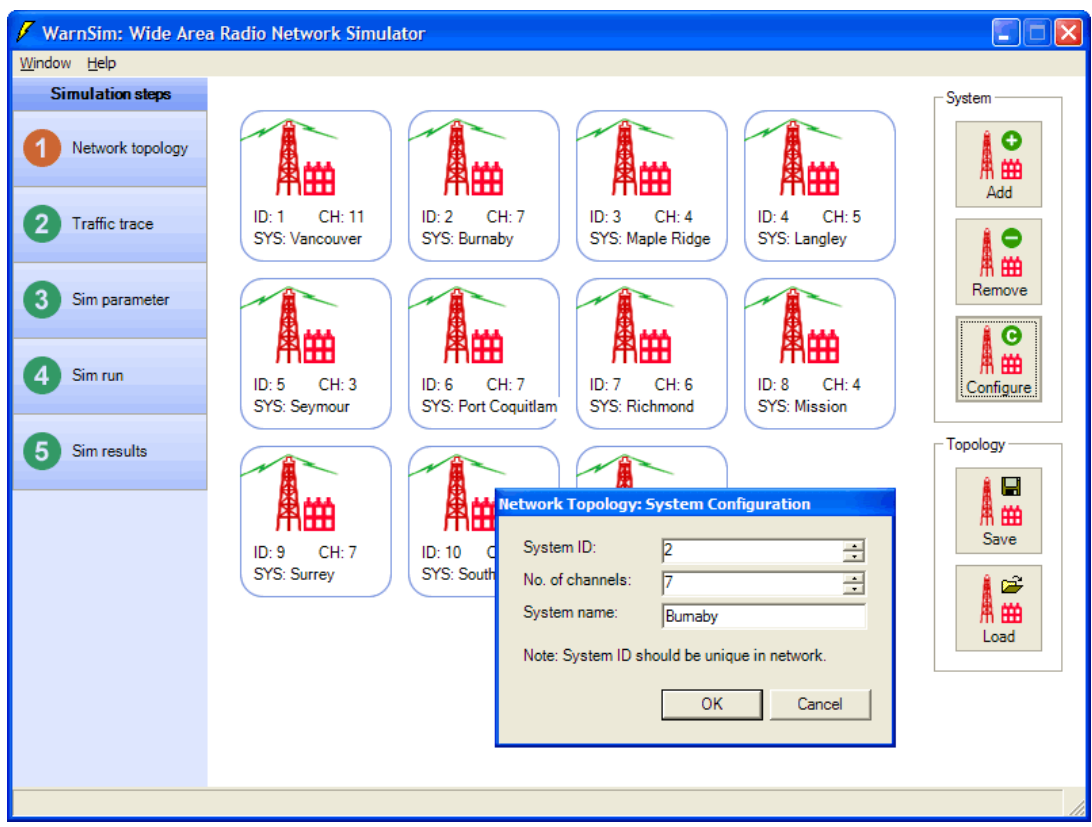

(b)

Figure 5.4: WarnSim: (a) network topology and (b) network configuration screen. 


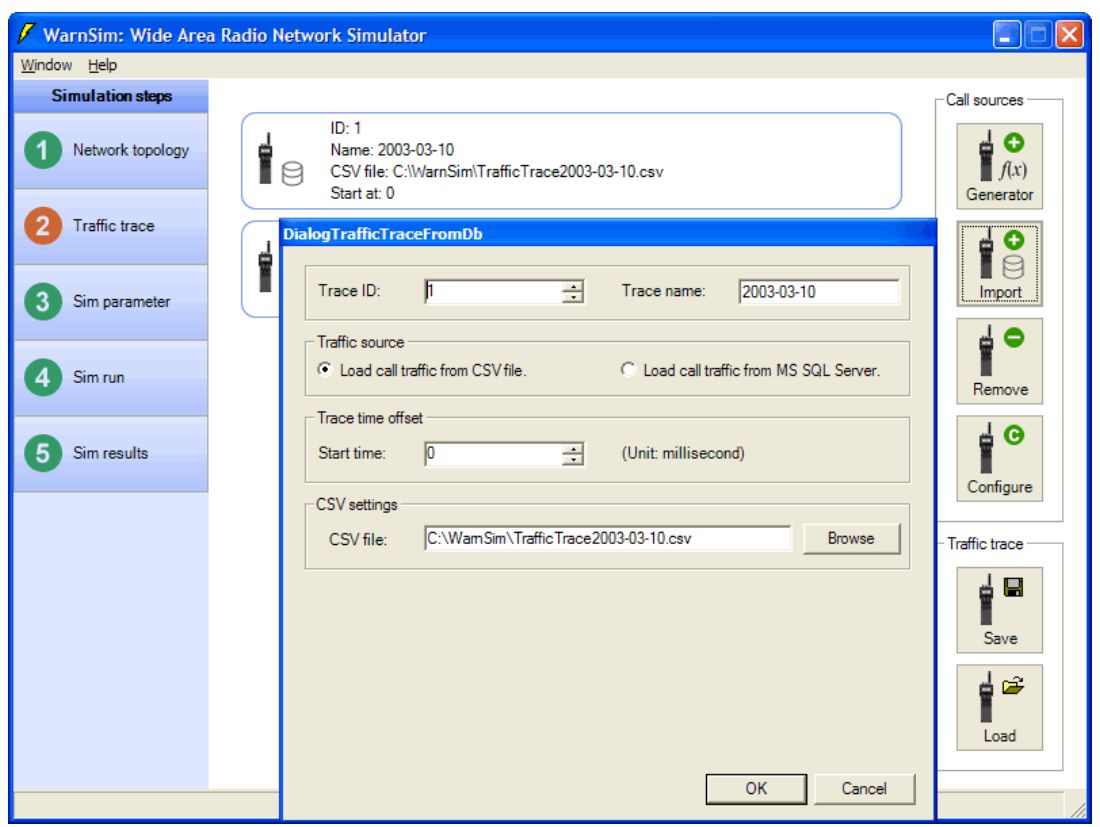

Figure 5.5: WarnSim traffic source configuration screen: importing traffic.

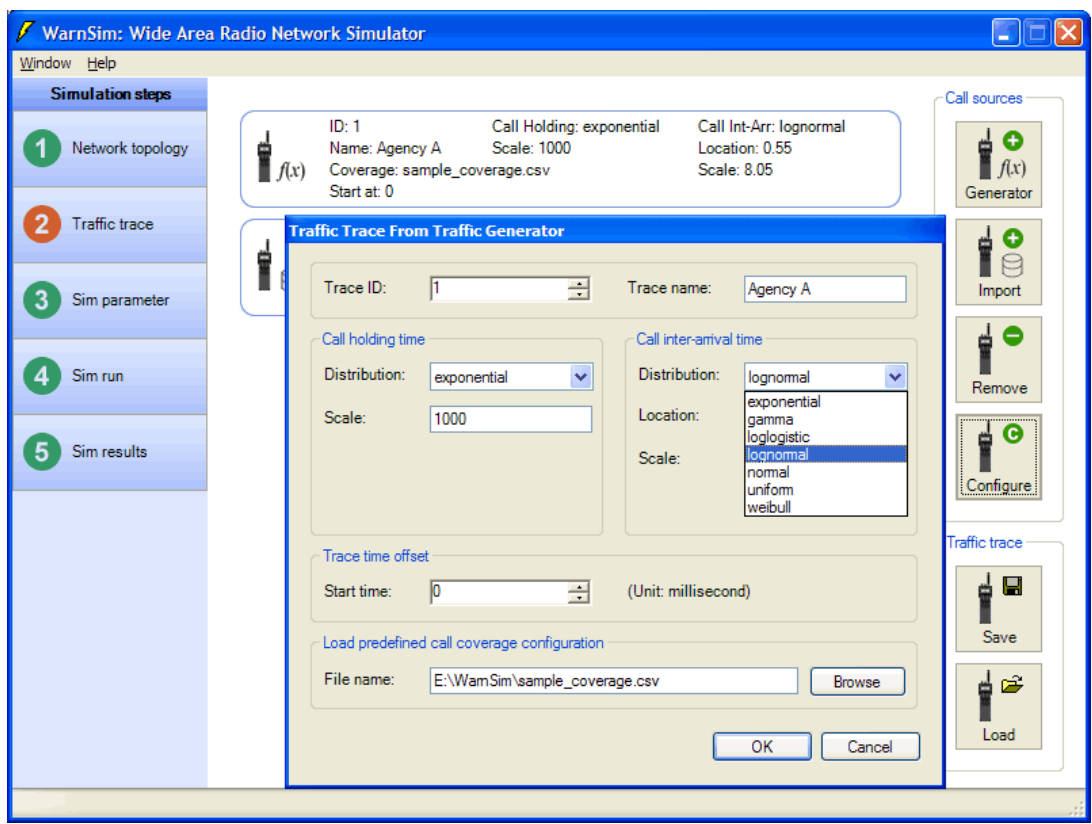

Figure 5.6: WarnSim traffic source configuration screen: generating traffic. 


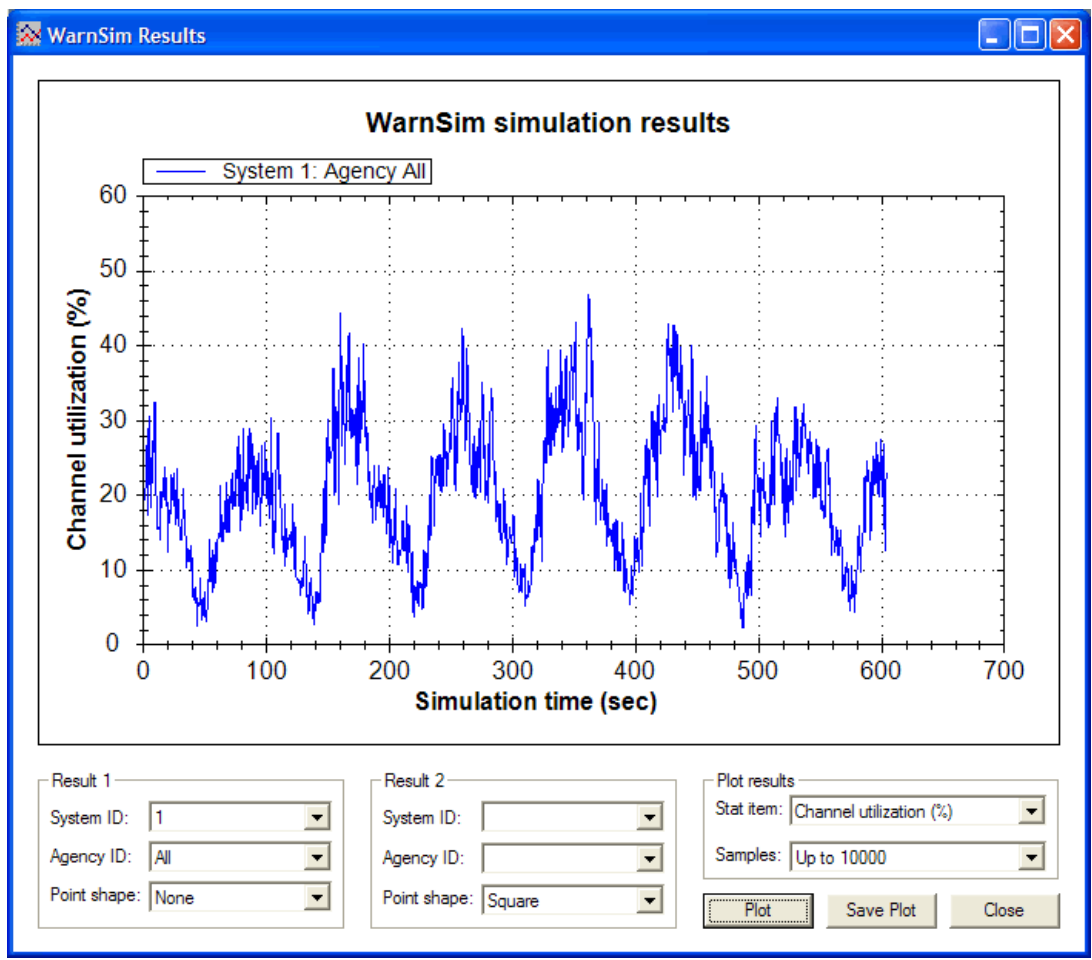

(a)

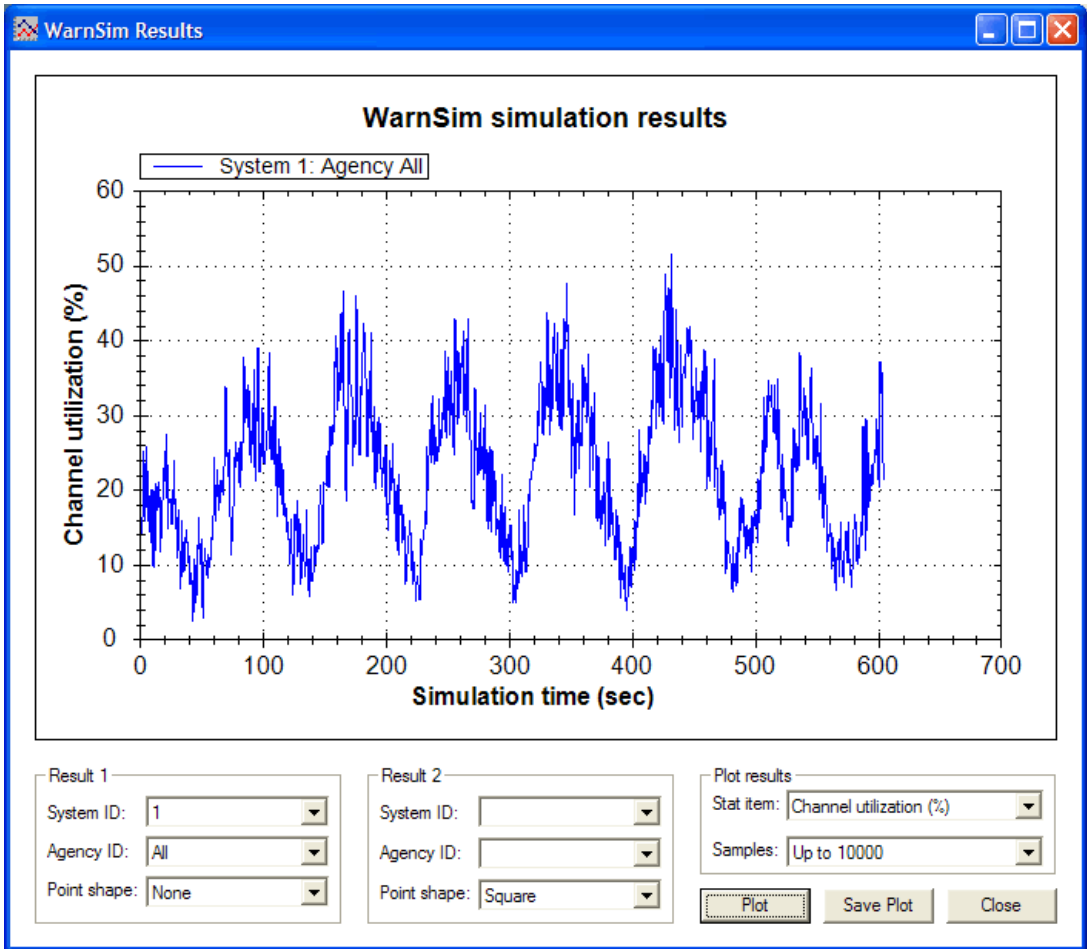

(b)

Figure 5.7: WarnSim simulation results screens for channel utilization of Vancouver system during: (a) a sample week in 2002 and (b) a sample week in 2003. The graphs show the running average calculated over ten-minute intervals. 


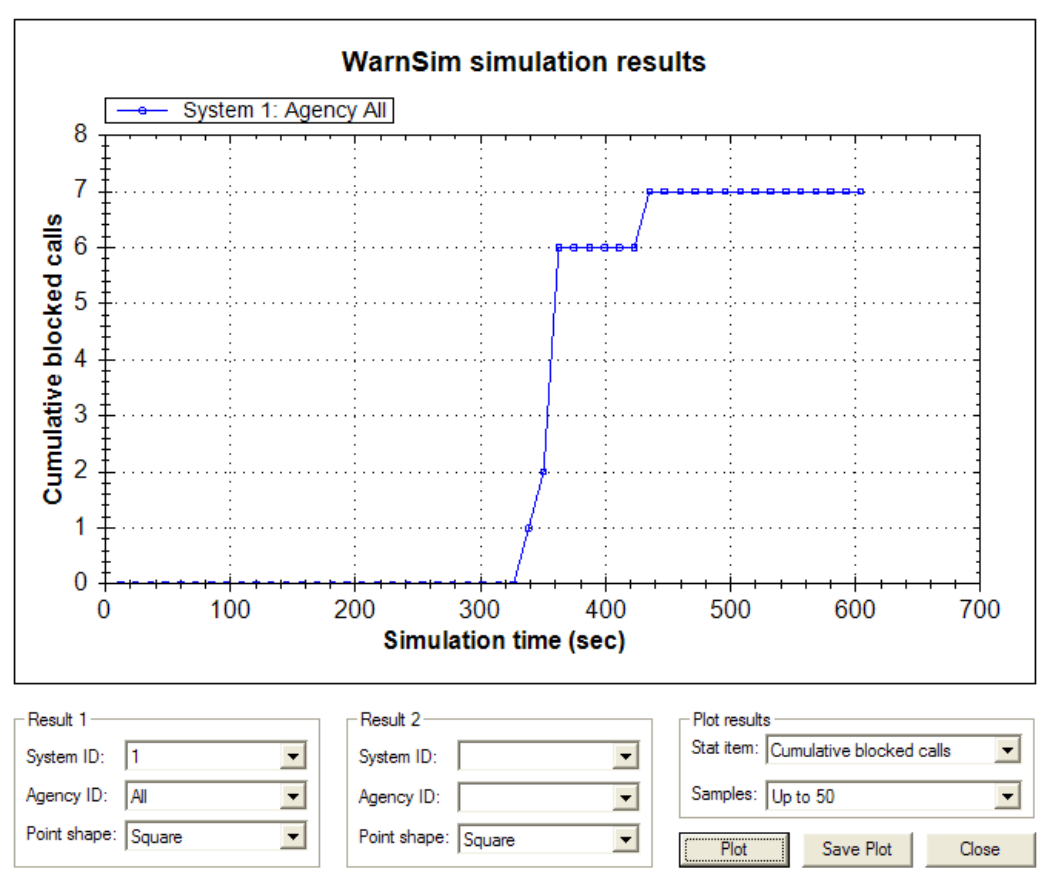

(a)

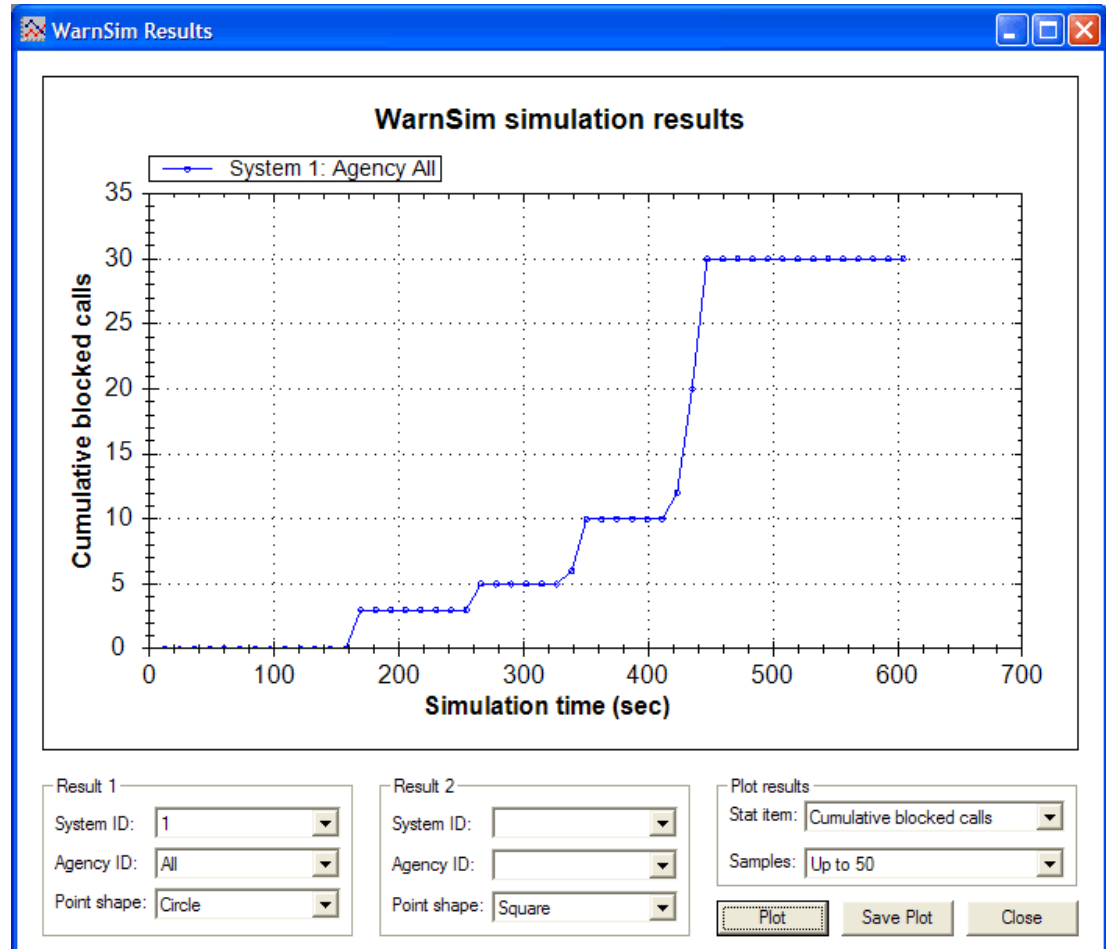

(b)

Figure 5.8: WarnSim simulation results screens for the cumulative number of blocked calls in Vancouver system during: (a) a sample week in 2002 and (b) a sample week in 2003. 


\subsection{Validation of WarnSim simulator}

We performed several steps to validate WarnSim. We first use build-in K-S GoF functions provided by MATLAB [39] and S-PLUS [40] to test the random variable generator implemented in WarnSim. All K-S GoF tests return high p-values $(>0.1)$, which validates the WarnSim generated random variables.

We then use various artificial testing traces to validate the deterministic WarnSim modules, such as call admission control module and simulation statistics module. A simple example of the testing scenario is to let 11 calls simultaneously reach a system with 10 channels. As expected, one call will be queued due to the lack of a free channel.

We further validate WarnSim by comparing the prediction results of Erlang B model with WarnSim simulation results. As shown in Table 5.1, call blocking probabilities match very well.

Table 5.1: Comparison of call blocking probability predicted by Erlang B model and the WarnSim simulated call blocking probability.

\begin{tabular}{|l|l|l|}
\hline & Erlang B model & WarnSim \\
\hline Network configuration & 10 phone lines & 1 system with 10 channels \\
\hline Call traffic volume & 10 Erlangs & 10 Erlangs \\
\hline Call holding time & exponentially distributed & $\begin{array}{l}\text { exponentially distributed with } \\
\text { mean value of } 180 \text { seconds }\end{array}$ \\
\hline Call inter-arrival time & exponentially distributed & $\begin{array}{l}\text { exponentially distributed with } \\
\text { mean value of } 18 \text { seconds }\end{array}$ \\
\hline $\begin{array}{l}\text { Dealing with blocked } \\
\text { calls }\end{array}$ & $\begin{array}{l}\text { blocked calls neither } \\
\text { queued nor retried }\end{array}$ & $\begin{array}{l}\text { Max Queuing Time }=0 ; \text { blocked } \\
\text { calls are not retried }\end{array}$ \\
\hline $\begin{array}{l}\text { Call blocking } \\
\text { probability }\end{array}$ & $21.5 \%$ & $\begin{array}{l}17 \%-27 \% ; \text { average }=21.86 \% \\
(10 \text { simulation runs })\end{array}$ \\
\hline
\end{tabular}




\subsection{Scalability and performance of WarnSim}

WarnSim provides good flexibility in choosing the network size (the number of cells in the network) and cell capacities (the number of channels in each cell). In our simulation experiments, WarnSim successfully handled sample networks with 100 cells and 50 channels in each cell. They are approximately ten times larger than the size of the E-Comm network and are sufficient to model any deployed PSWNs.

WarnSim simulation tests are performed on a windows workstation equipped with Intel Pentium 4 2.8GHz CPU and 512Mbyte memory. The operating system is Microsoft Windows XP Professional with .NET framework 1.1 supports. On this platform, a simulation with one hour of busy hour traffic from the E-Comm PSWN lasts less than one minute. The high performance of WarnSim enables users to quickly test various network configurations and call traffic, and to efficiently perform trace driven simulations and evaluate network performances during busy hours. 


\section{CHAPTER 6: SIMULATION AND PREDICTION}

In this Chapter, we use WarnSim to validate the proposed traffic model and to evaluate and predict E-Comm network performance.

\subsection{Validation of the proposed traffic model}

To validate the proposed traffic model, we first use WarnSim to generate traffic traces and compare them with the collected E-Comm traffic data. WarnSim distribution parameters listed in Table 6.1 are used for the call generator to model busy hour traffic of the 2003 dataset.

Table 6.1: Distribution parameters for the WarnSim call generator.

\begin{tabular}{|l|l|l|l|}
\hline & Agency A & Agency B & Agency C \\
\hline Call holding time & Lognormal & lognormal & lognormal \\
& $\sigma=8.05$ & $\sigma=8.09$ & $\sigma=7.88$ \\
& $\mu=0.55$ & $\mu=0.73$ & $\mu=0.82$ \\
\hline Call inter-arrival time & Exponential & exponential & exponential \\
& $\beta=1354$ & $\beta=761$ & $\beta=3480$ \\
\hline
\end{tabular}

In the simulation scenarios, systems and channels shown in Table 6.2 reflect the configuration of the E-Comm network during the busiest hour in 2003.

Table 6.2: System IDs and number of channels.

\begin{tabular}{|c|c|c|c|c|c|c|c|c|c|c|c|}
\hline System ID & 1 & 2 & 3 & 4 & 5 & 6 & 7 & 8 & 9 & 10 & 11 \\
\hline Channels & 10 & 7 & 4 & 5 & 3 & 7 & 8 & 4 & 7 & 6 & 3 \\
\hline
\end{tabular}


Simulation results listed in Table 6.3 indicate that the proposed traffic model performs adequately when used to evaluate call blocking probability and channel utilization in the E-Comm network.

Table 6.3: Comparison of network performance: using collected traffic (actual) vs. model generated traffic (simulated).

\begin{tabular}{|c|c|c|c|c|}
\hline System ID & $\begin{array}{c}\text { Actual } \\
\text { blocking } \\
\text { probability } \\
(\%)\end{array}$ & $\begin{array}{c}\text { Actual } \\
\text { channel } \\
\text { utilization } \\
(\%)\end{array}$ & $\begin{array}{c}\text { Simulated } \\
\text { blocking } \\
\text { probability } \\
(\%)\end{array}$ & $\begin{array}{c}\text { Simulated } \\
\text { channel } \\
\text { utilization } \\
(\%)\end{array}$ \\
\hline 1 & $1.9-3.5$ & $57-65$ & $2.9-3.9$ & $53-56$ \\
\hline 2 & $0.0-0.6$ & $29-48$ & $0.8-1.1$ & $34-37$ \\
\hline 3 & 0.0 & $11-14$ & 0.0 & 11 \\
\hline 4 & $0.0-0.4$ & $21-23$ & $0.4-1.3$ & $21-26$ \\
\hline 5 & 0.0 & $4-17$ & $0.0-1.1$ & $10-11$ \\
\hline 6 & $0.0-0.3$ & $19-42$ & $0.1-0.3$ & $27-29$ \\
\hline 7 & $0.0-0.4$ & $25-34$ & $0.0-0.2$ & $25-27$ \\
\hline 8 & 0.0 & $8-11$ & 0.0 & $9-10$ \\
\hline 9 & $0.3-0.5$ & $37-43$ & $1.1-2.0$ & $36-39$ \\
\hline 10 & 0.0 & $16-26$ & $0.1-0.2$ & $20-22$ \\
\hline 11 & 0.0 & $6-10$ & 0.0 & $6-8$ \\
\hline
\end{tabular}

\subsection{Evaluation of E-Comm network performance}

We can evaluate the Grade of Service (GoS) of PSWN by performing WarnSim simulations using genuine traffic data. In this Section, we first describe the simulation scenarios and source traffic traces. We then describe the evaluation results of two GoS standards in the E-Comm network: call blocking probability and channel utilization.

\subsubsection{Network performance during busy hours}

Three simulation scenarios are setup to evaluate the GoS of the E-Comm network during the top three busiest hours in 2003. Systems and channels reflect the 
corresponding configurations of the E-Comm network, as shown in Table 6.4. The source traffic traces are extracted from the top three busiest hours in 2003 dataset and are transformed into the format shown in Table 3.1. Maximum queuing time of blocked call is set to zero. Simulation statistics including channel utilization, total number of calls, number of blocked calls, and call blocking probability for each system are collected by WarnSim. Simulation results shown in Tables 6.5-6.7 indicate that System 1 is the busiest system in the E-Comm network in terms of both hourly call arrival rate and call blocking probability. Few calls may be blocked in Systems 2, 4, 6, 7, and 9. In other systems, calls never got blocked and channel utilizations are relatively low.

Table 6.4: System IDs and number of channels during the top three busy hours in the 2003 dataset.

\begin{tabular}{|c|c|c|c|}
\hline System ID & $\begin{array}{c}\text { No. of } \\
\text { channels in } \\
\text { busy hour 1 }\end{array}$ & $\begin{array}{c}\text { No. of } \\
\text { channels in } \\
\text { busy hour 2 }\end{array}$ & $\begin{array}{c}\text { No. of } \\
\text { channels in } \\
\text { busy hour 3 }\end{array}$ \\
\hline 1 & 10 & 10 & 10 \\
\hline 2 & 7 & 7 & 7 \\
\hline 3 & 4 & 4 & 4 \\
\hline 4 & 5 & 5 & 5 \\
\hline 5 & 3 & 3 & 3 \\
\hline 6 & 7 & 7 & 7 \\
\hline 7 & 8 & 8 & 7 \\
\hline 8 & 4 & 4 & 4 \\
\hline 9 & 7 & 7 & 7 \\
\hline 10 & 6 & 6 & 6 \\
\hline 11 & 3 & 3 & 3 \\
\hline
\end{tabular}


Table 6.5: Simulation results of the busiest hour in the 2003 dataset.

\begin{tabular}{|c|r|r|r|r|}
\hline System ID & $\begin{array}{c}\text { Channel } \\
\text { utilization }\end{array}$ & $\begin{array}{c}\text { Total No. of } \\
\text { calls }\end{array}$ & $\begin{array}{c}\text { No. of } \\
\text { blocked calls }\end{array}$ & $\begin{array}{c}\text { Blocking } \\
\text { Probability }\end{array}$ \\
\hline 1 & $57 \%$ & 5,070 & 95 & $1.9 \%$ \\
\hline 2 & $48 \%$ & 2,907 & 16 & $0.6 \%$ \\
\hline 3 & $13 \%$ & 426 & 0 & $0.0 \%$ \\
\hline 4 & $23 \%$ & 1,133 & 5 & $0.4 \%$ \\
\hline 5 & $17 \%$ & 490 & 0 & $0.0 \%$ \\
\hline 6 & $42 \%$ & 2,602 & 9 & $0.3 \%$ \\
\hline 7 & $31 \%$ & 2,150 & 0 & $0.0 \%$ \\
\hline 8 & $11 \%$ & 391 & 0 & $0.0 \%$ \\
\hline 9 & $43 \%$ & 2,804 & 12 & $0.4 \%$ \\
\hline 10 & $26 \%$ & 1,368 & 0 & $0.0 \%$ \\
\hline 11 & $10 \%$ & 301 & 0 & $0.0 \%$ \\
\hline
\end{tabular}

Table 6.6: Simulation results of the second busiest hour in the 2003 dataset.

\begin{tabular}{|c|r|r|r|r|}
\hline System ID & $\begin{array}{c}\text { Channel } \\
\text { utilization }\end{array}$ & $\begin{array}{c}\text { Total No. of } \\
\text { calls }\end{array}$ & $\begin{array}{c}\text { No. of } \\
\text { blocked calls }\end{array}$ & $\begin{array}{c}\text { Blocking } \\
\text { Probability }\end{array}$ \\
\hline 1 & $65 \%$ & 5,790 & 205 & $3.5 \%$ \\
\hline 2 & $29 \%$ & 1,676 & 0 & $0.0 \%$ \\
\hline 3 & $14 \%$ & 491 & 0 & $0.0 \%$ \\
\hline 4 & $21 \%$ & 949 & 0 & $0.0 \%$ \\
\hline 5 & $7 \%$ & 197 & 0 & $0.0 \%$ \\
\hline 6 & $19 \%$ & 1,185 & 0 & $0.0 \%$ \\
\hline 7 & $25 \%$ & 1,773 & 0 & $0.0 \%$ \\
\hline 8 & $9 \%$ & 324 & 0 & $0.0 \%$ \\
\hline 9 & $37 \%$ & 2,453 & 12 & $0.5 \%$ \\
\hline 10 & $22 \%$ & 1,202 & 0 & $0.0 \%$ \\
\hline 11 & $7 \%$ & 204 & 0 & $0.0 \%$ \\
\hline
\end{tabular}


Table 6.7: Simulation results of the third busiest hour in 2003 dataset.

\begin{tabular}{|c|r|r|r|r|}
\hline System ID & $\begin{array}{r}\text { Channel } \\
\text { utilization }\end{array}$ & $\begin{array}{c}\text { Total No. of } \\
\text { calls }\end{array}$ & $\begin{array}{c}\text { No. of } \\
\text { blocked calls }\end{array}$ & $\begin{array}{c}\text { Blocking } \\
\text { probability }\end{array}$ \\
\hline 1 & $60 \%$ & 5,484 & 169 & $3.0 \%$ \\
\hline 2 & $34 \%$ & 2,131 & 1 & $0.0 \%$ \\
\hline 3 & $11 \%$ & 459 & 0 & $0.0 \%$ \\
\hline 4 & $21 \%$ & 1,045 & 0 & $0.0 \%$ \\
\hline 5 & $4 \%$ & 106 & 0 & $0.0 \%$ \\
\hline 6 & $25 \%$ & 1,677 & 1 & $0.1 \%$ \\
\hline 7 & $34 \%$ & 2,141 & 8 & $0.4 \%$ \\
\hline 8 & $8 \%$ & 353 & 0 & $0.0 \%$ \\
\hline 9 & $37 \%$ & 2,316 & 8 & $0.3 \%$ \\
\hline 10 & $16 \%$ & 960 & 0 & $0.0 \%$ \\
\hline 11 & $6 \%$ & 178 & 0 & $0.0 \%$ \\
\hline
\end{tabular}

\subsubsection{Number of channels and Grade of Service}

We now investigate the relationship between the number of available channels and the GoS (channel utilization and call blocking probability). The second busiest hour in 2003 dataset is chosen because it has the highest call blocking probability in System 1 among the top three busiest hours in the 2003 dataset. While keeping the number of channels in the remaining systems unchanged, we vary the number of available channels in System 1 from 1 to 14. WarnSim simulation results are collected from System 1. In this scenario, the maximum queuing time of blocked calls is set to zero.

Simulation results shown in Table 6.8 and Figure 6.1 depict the relationship between the number of channels and the call blocking probability. When the number of channels increases from 1 to 8 , call blocking probability drops to $12 \%$ at a near constant rate as shown in Figure 6.1. It then decreases slowly to zero when the number of channels reaches 14 . The channel utilization drops slowly when the number of channels increases 
from 1 to 8 and then it drops at a constant rate. Based on these results, we can determine the number of necessary channels in order to meet a certain Grade of Service.

Table 6.8: Relationship between number of channels, call blocking probability, and channel utilization. Maximum queuing time is set to zero.

\begin{tabular}{|c|c|c|}
\hline No. of channels & $\begin{array}{c}\text { Channel utilization } \\
(\%)\end{array}$ & $\begin{array}{l}\text { Blocking probability } \\
(\%)\end{array}$ \\
\hline 1 & 88 & 86.5 \\
\hline 2 & 87 & 73.6 \\
\hline 3 & 87 & 61.0 \\
\hline 4 & 85 & 49.5 \\
\hline 5 & 83 & 38.0 \\
\hline 6 & 81 & 27.9 \\
\hline 7 & 78 & 19.5 \\
\hline 8 & 74 & 12.3 \\
\hline 9 & 69 & 6.8 \\
\hline 10 & 64 & 3.4 \\
\hline 11 & 59 & 1.3 \\
\hline 12 & 54 & 0.4 \\
\hline 13 & 50 & 0.1 \\
\hline 14 & 46 & 0.0 \\
\hline
\end{tabular}




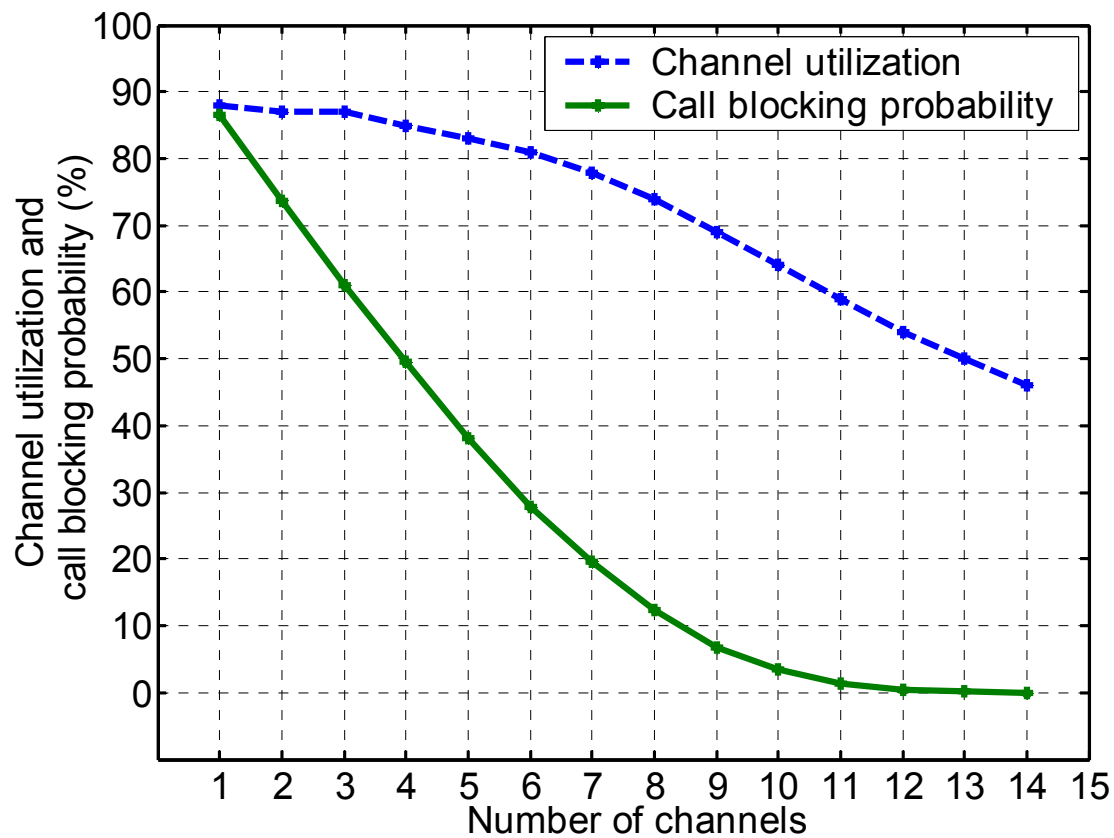

Figure 6.1: Relationship between number of channels, call blocking probability, and channel utilization. Maximum queuing time is set to zero.

\subsubsection{Maximum queuing time and Grade of Service}

An effective way to lower the call blocking probability without adding new channels is using call queuing mechanism. The call queuing mechanism implies that instead of dropping a call immediately after it failed to obtain a free channel, the call is placed in a waiting queue for a certain amount of time. The call is dropped if it cannot get the required channels after waiting for the amount of time specified by the maximum queuing time.

We simulate scenarios where maximum queuing time increases from 0 to 1.5 seconds and collect call blocking probability statistics. The second busiest hour in the 2003 dataset is chosen for simulation. Simulation results shown in Table 6.9 and Figure 6.2 indicate that increasing the maximum queuing time can effectively lower the call 
blocking probability. The call blocking probability of System 1 is lowered by more than $80 \%$ when the maximum queuing time is increased from 0 to 1 second.

Table 6.9: Maximum call queuing time vs. call blocking probability.

\begin{tabular}{|c|c|}
\hline $\begin{array}{c}\text { Maximum queuing time } \\
(\mathrm{ms})\end{array}$ & $\begin{array}{c}\text { Call blocking probability } \\
(\%)\end{array}$ \\
\hline 0 & 3.4 \\
\hline 100 & 2.7 \\
\hline 200 & 2.3 \\
\hline 300 & 1.8 \\
\hline 400 & 1.6 \\
\hline 500 & 1.4 \\
\hline 600 & 1.0 \\
\hline 700 & 0.9 \\
\hline 800 & 0.7 \\
\hline 900 & 0.6 \\
\hline 1,000 & 0.5 \\
\hline 1,100 & 0.4 \\
\hline 1,200 & 0.3 \\
\hline 1,300 & 0.3 \\
\hline 1,400 & 0.3 \\
\hline 1,500 & 0.2 \\
\hline
\end{tabular}




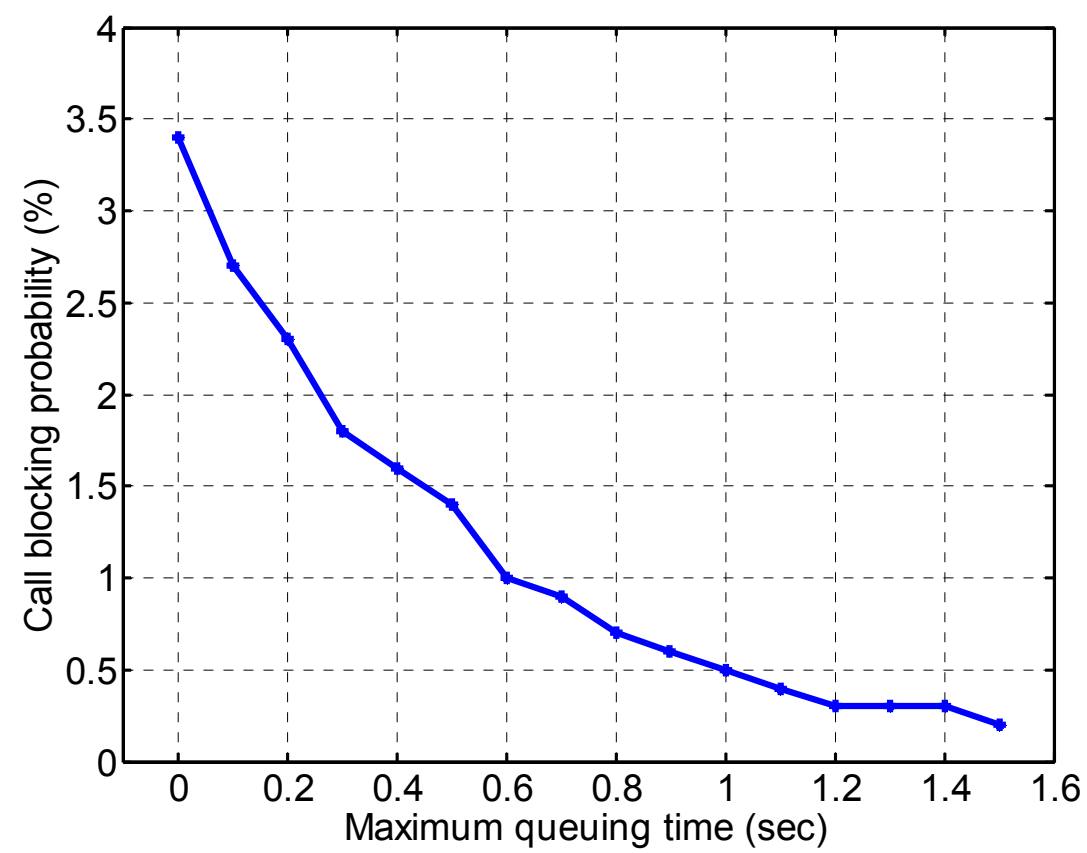

Figure 6.2: Maximum call queuing time vs. call blocking probability.

\subsection{Prediction of the E-Comm network performance}

In this Section, we predict the E-Comm network performance in terms of call blocking probability in cases of increased call traffic volume. Two case scenarios are investigated. The system IDs and the number of channels for both scenarios are shown in Table 6.10. The maximum queuing time in both scenarios is set to zero.

Table 6.10: System IDs and number of channels.

\begin{tabular}{|c|c|c|c|c|c|c|c|c|c|c|c|}
\hline System ID & 1 & 2 & 3 & 4 & 5 & 6 & 7 & 8 & 9 & 10 & 11 \\
\hline Channels & 10 & 7 & 4 & 5 & 3 & 7 & 8 & 4 & 7 & 6 & 3 \\
\hline
\end{tabular}

\subsubsection{Simulation scenario one}

We first consider a sample scenario where the number of calls made by Agency A increases by $100 \%$ from the 2003 busy hours. WarnSim distribution parameters for the call generator are listed in Table 6.11. 
Table 6.11: Scenario one: distribution parameters for the WarnSim call generator.

\begin{tabular}{|l|l|l|l|}
\hline & Agency A & Agency B & Agency C \\
\hline Call holding time & Lognormal & lognormal & lognormal \\
& $\sigma=8.05$ & $\sigma=8.09$ & $\sigma=7.88$ \\
& $\mu=0.55$ & $\mu=0.73$ & $\mu=0.82$ \\
\hline Call inter-arrival time & Exponential & exponential & exponential \\
& $\beta=677$ & $\beta=761$ & $\beta=3480$ \\
\hline
\end{tabular}

The simulation results shown in Table 6.12 indicate that the call blocking probability of Systems 1 visibly increases after the call traffic increase.

Table 6.12: Comparison of network performance. Scenario one: using collected traffic (original) vs. model generated traffic after the increase (predicted). Cells with call blocking probability higher than $3 \%$ are marked in grey.

\begin{tabular}{|c|c|c|c|c|}
\hline System ID & $\begin{array}{c}\text { Original } \\
\text { blocking } \\
\text { probability } \\
(\%)\end{array}$ & $\begin{array}{c}\text { Original } \\
\text { channel } \\
\text { utilization } \\
(\%)\end{array}$ & $\begin{array}{c}\text { Predicted } \\
\text { blocking } \\
\text { probability } \\
(\%)\end{array}$ & $\begin{array}{c}\text { Predicted } \\
\text { channel } \\
\text { utilization } \\
(\%)\end{array}$ \\
\hline 1 & $1.9-3.5$ & $57-65$ & $12.5-13.5$ & $71-72$ \\
\hline 2 & $0.0-0.6$ & $29-48$ & $0.8-1.1$ & $35-37$ \\
\hline 3 & 0.0 & $11-14$ & 0.0 & $11-12$ \\
\hline 4 & $0.0-0.4$ & $21-23$ & $0.1-0.2$ & 22 \\
\hline 5 & 0.0 & $4-17$ & $0.0-1.8$ & $7-11$ \\
\hline 6 & $0.0-0.3$ & $19-42$ & $0.3-0.5$ & $28-31$ \\
\hline 7 & $0.0-0.4$ & $25-34$ & $0.0-0.1$ & $28-30$ \\
\hline 8 & 0.0 & $8-11$ & 0.0 & 10 \\
\hline 9 & $0.3-0.5$ & $37-43$ & $1.3-2.0$ & $39-40$ \\
\hline 10 & 0.0 & $16-26$ & $0.0-0.1$ & $21-22$ \\
\hline 11 & 0.0 & $6-10$ & $0.0-0.8$ & $7-8$ \\
\hline
\end{tabular}

Further WarnSim simulations reveal that System 1 requires 4 additional channels in order to maintain call blocking probabilities below the required 3\% [7]. 


\subsubsection{Simulation scenario two}

We now consider the sample scenario where the number of calls made by Agency B increases by $100 \%$ from 2003 busy hours. WarnSim distribution parameters for the call generator are listed in Table 6.13.

Table 6.13: Scenario two: distribution parameters for the WarnSim call generator.

\begin{tabular}{|l|l|l|l|}
\hline & Agency A & Agency B & Agency C \\
\hline Call holding time & Lognormal & lognormal & lognormal \\
& $\sigma=8.05$ & $\sigma=8.09$ & $\sigma=7.88$ \\
& $\mu=0.55$ & $\mu=0.73$ & $\mu=0.82$ \\
\hline Call inter-arrival time & exponential & exponential & exponential \\
& $\beta=1354$ & $\beta=381$ & $\beta=3480$ \\
\hline
\end{tabular}

The simulation results shown in Table 6.14 indicate that the call blocking probabilities of four systems (IDs 1, 2, 4, and 9) visibly increase after the call traffic increase.

Further simulations reveal that in order to maintain call blocking probabilities of all systems below 3\%, Systems 1, 2, 4, and 9 require 4, 2, 1, and 3 additional channels, respectively. 
Table 6.14: Comparison of network performance. Scenario two: using collected traffic (original) vs. model generated traffic after the increase (predicted). Cells with call blocking probability higher than $3 \%$ are marked in grey.

\begin{tabular}{|c|c|c|c|c|}
\hline System ID & $\begin{array}{c}\text { Original } \\
\text { blocking } \\
\text { probability } \\
(\%)\end{array}$ & $\begin{array}{c}\text { Original } \\
\text { channel } \\
\text { utilization } \\
(\%)\end{array}$ & $\begin{array}{c}\text { Predicted } \\
\text { blocking } \\
\text { probability } \\
(\%)\end{array}$ & $\begin{array}{c}\text { Predicted } \\
\text { channel } \\
\text { utilization } \\
(\%)\end{array}$ \\
\hline 1 & $1.9-3.5$ & $57-65$ & $12.1-12.6$ & $71-72$ \\
\hline 2 & $0.0-0.6$ & $29-48$ & $5.1-7.0$ & $54-55$ \\
\hline 3 & 0.0 & $11-14$ & $0.1-0.4$ & $16-17$ \\
\hline 4 & $0.0-0.4$ & $21-23$ & $1.5-3.7$ & $35-39$ \\
\hline 5 & 0.0 & $4-17$ & $1.0-1.3$ & $16-18$ \\
\hline 6 & $0.0-0.3$ & $19-42$ & $2.1-2.7$ & $44-45$ \\
\hline 7 & $0.0-0.4$ & $25-34$ & $0.6-0.8$ & $38-40$ \\
\hline 8 & 0.0 & $8-11$ & $0.0-0.3$ & $16-18$ \\
\hline 9 & $0.3-0.5$ & $37-43$ & $9.0-10.1$ & $60-62$ \\
\hline 10 & 0.0 & $16-26$ & $1.3-1.5$ & $35-38$ \\
\hline 11 & 0.0 & $6-10$ & $0.3-0.9$ & $11-13$ \\
\hline
\end{tabular}




\section{CHAPTER 7: CONCLUSIONS AND FUTURE WORK}

In this Chapter, we conclude this thesis and describe possible future work.

\subsection{Conclusions and contributions}

In this thesis, we described the structure of the E-Comm network and the collection of traffic data. We then analyzed the E-Comm traffic data and characterized its properties such as the daily and weekly cyclic patterns of call arrival rate and call holding time. We proposed a statistical model of call traffic on the user agency level. The lognormal distribution proved to be appropriate for modeling call holding time, while the exponential distribution was suitable for modeling call inter-arrival time. We also addressed the call coverage issue of group calls.

In order to evaluate and predict the performance of the E-Comm network, we developed a new simulation tool named WarnSim. With WarnSim simulations, we validated the proposed traffic model and evaluated the performance of the E-Comm network. We found that the E-Comm network is capable of handling busy hour traffic based on the 2002 and the 2003 E-Comm traffic data. We predicted E-Comm network performance in cases of increased call traffic volume. Simulation results showed that when call traffic generated by E-Comm heavy user agencies increases by $100 \%$ during busy hours, the E-Comm network was unable to maintain the required Grade of Service. Further WarnSim simulations suggested the E-Comm network capacity (number of channels in each system) needed to accommodate the increased call traffic. 


\subsection{Future work}

The related network traffic prediction model, such as the cluster-based SARIMA model developed by H. Chen [41], can be adopted to work with the proposed traffic model and the WarnSim simulator to better predict future performance of the E-Comm network.

WarnSim simulator may be improved and optimized. There are seven types of random variables currently available in WarnSim. Additional random variables may be added to WarnSim when needs arise. Furthermore, the internal algorithm of WarnSim may be further optimized to achieve better performance in terms of simulation speed.

Ad hoc networks are self-organizing networks of user terminals that form without the need for prior network infrastructure. Because of their adaptable, robust, and rapidly deployable potentials, ad hoc networks are being considered for the next generation PSWNs. In [42], D. Sharp proposed a multi-level hierarchical PSWN architecture that uses a dominating-set based backbone network to interconnect geographically distributed ad hoc networks in order to meet the needs of emergency communications. WarnSim could be further developed and adopted to evaluate performance of the new generation public safety wireless networks. 


\section{APPENDIX A: MAXIMUM LIKELIHOOD ESTIMATION (MLE) SCRIPTS}

\section{A.1 MLE script for lognormal distribution}

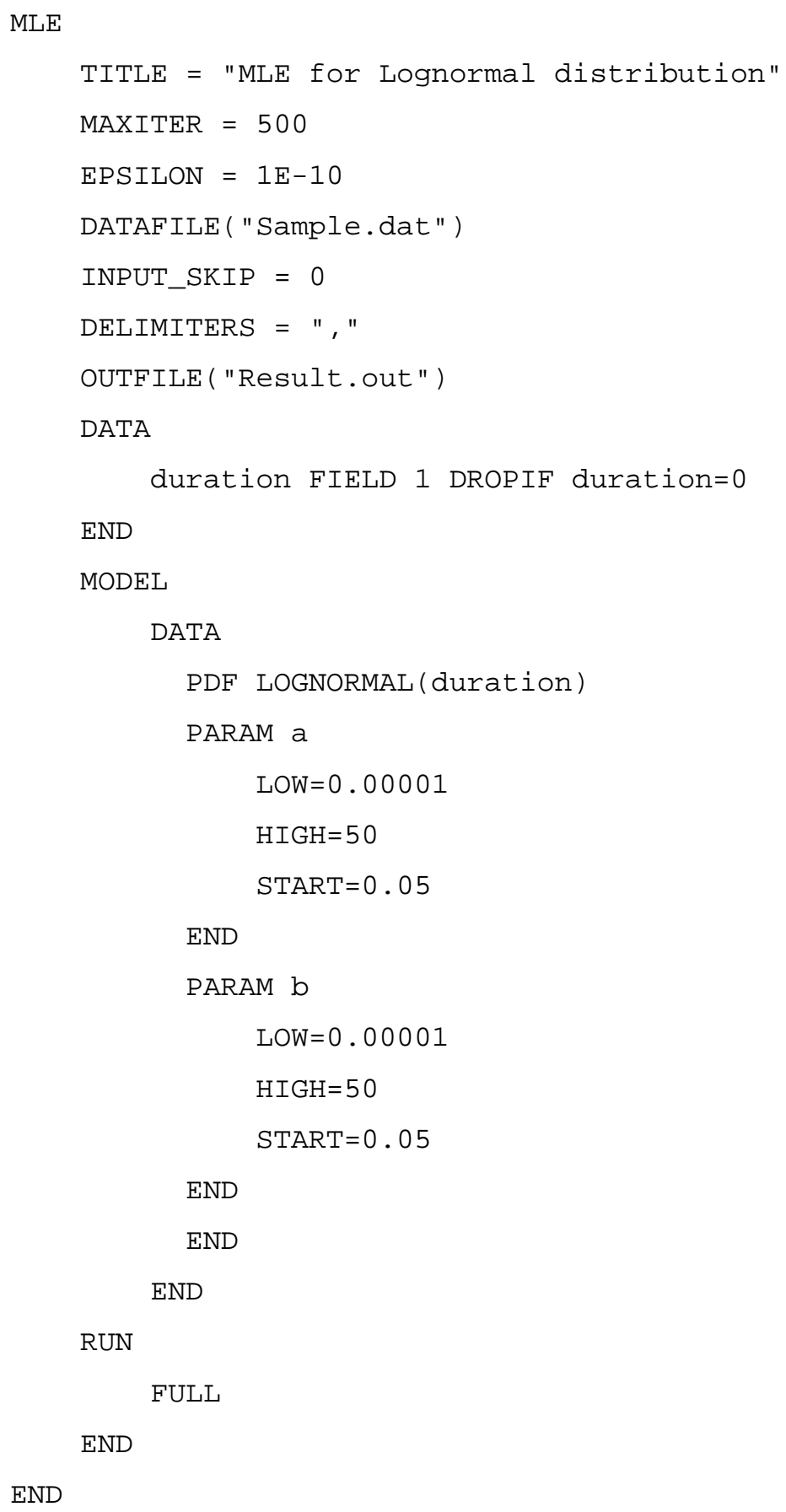




\section{A.2 MLE script for gamma distribution}

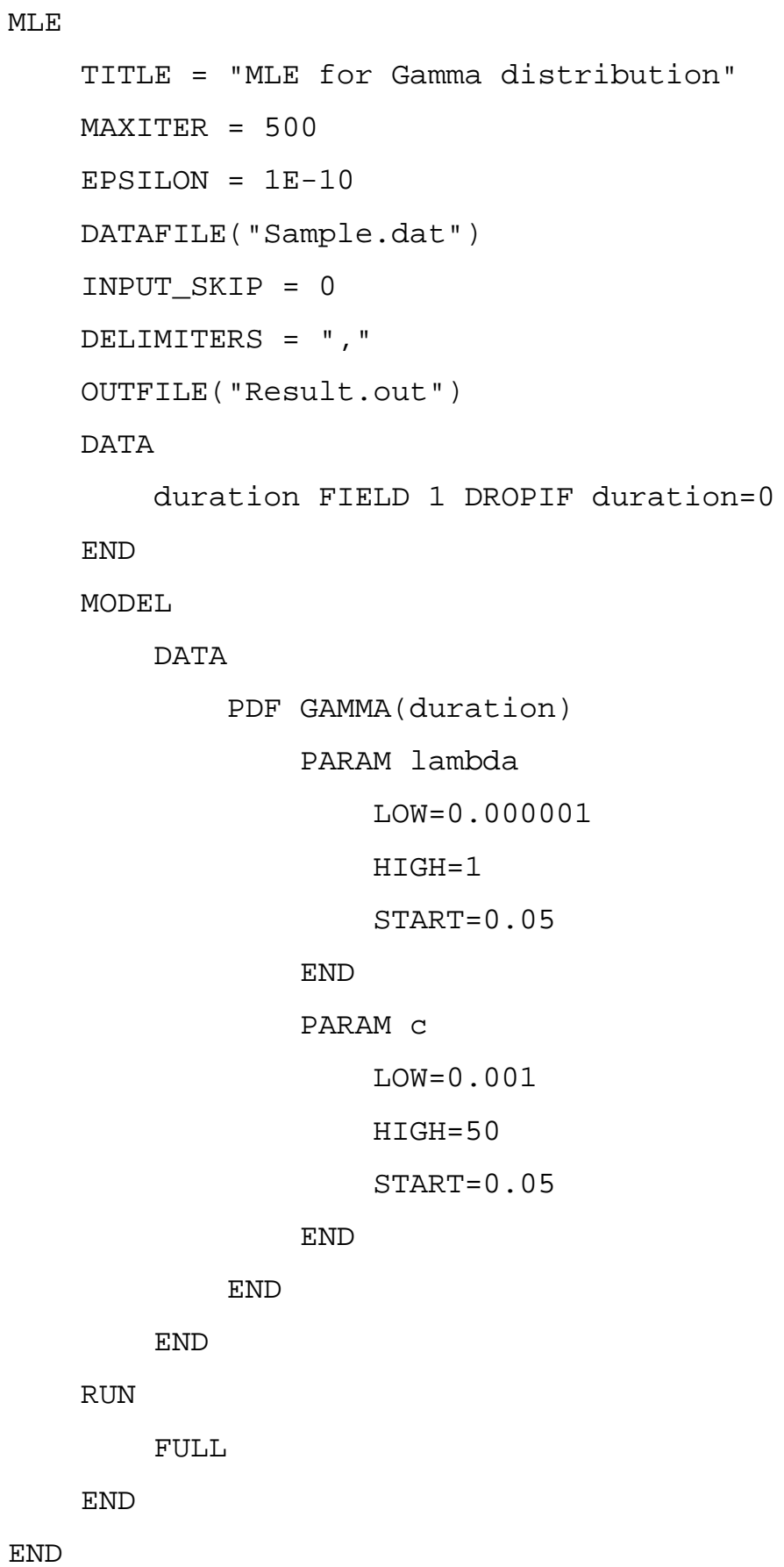




\section{REFERENCES}

[1] SAFECOM Program. Public Safety Communications and Interoperability Statement of Requirements [Online]. Available: http://www.safecomprogram.gov/ files/PSCI_Statement_of_Requirements_v1_0.pdf. Accessed: Aug. 15, 2004.

[2] SAFECOM Program. About SAFECOM [Online]. Available: http://www. safecomprogram.gov.

[3] Emergency Communications for Southwest British Columbia Incorporated [Online]. Available: http://www.ecomm.bc.ca.

[4] K. Bayne, 2000 operating budget: E-Comm and the Vancouver emergency [Online]. Available: http://www.city.vancouver.bc.ca/ctyclerk/cclerk/000420/ Csb1-4.htm. Accessed: Aug. 15, 2004.

[5] ComNet Ericsson. EDACS explained [Online]. Available: http://www. trunkedradio.net/trunked/edacs/EDACS_Whitepaper.pdf. Accessed: Aug. 15, 2004.

[6] The MathWorks. Fourier analysis and the Fast Fourier Transform (FFT) [Online]. Available: http://www.mathworks.com/access/helpdesk/help/techdoc/ math/datafun20.html. Accessed: Aug. 15, 2004.

[7] Industry Canada. Channel Loading Guidelines [Online]. Available: http://strategis.ic.gc.ca/epic/internet/insmt-gst.nsf/en/sf08015e.html. Accessed: Aug. 15, 2004.

[8] A. M. Law and W. D. Kelton, Simulation Modeling and Analysis, $3^{\text {rd }}$ ed. Toronto, ON: McGraw-Hill, 2000, p. 4.

[9] W. Gilchrist, Statistical Modelling. Toronto, ON: John Wiley \& Sons, 1984, pp. 194-205.

[10] Diagnostic Strategies. Traffic modeling and resource allocation in call centers [Online]. Available: http://www.diagnosticstrategies.com/traffic_modeling.htm. Accessed: Aug. 15, 2004.

[11] S. M. Ross, Simulation, $2^{\text {nd }}$ ed. Toronto, ON: Academic Press, 1997, pp. 189-202.

[12] D. J. Holman, Mle: a programming language for building likelihood models [Online]. Available: http://faculty.washington.edu/ djholman $/ \mathrm{mle}$. Accessed: Aug. 15, 2004.

[13] NIST/SEMATECH. NIST/SEMATECH e-Handbook of statistical methods [Online]. Available:http://www.itl.nist.gov/div898/handbook/toolaids/pff/eda.pdf. Accessed: Aug. 15, 2004. 
[14] V. A. Bolotin, "Modeling call holding time distributions for CCS network design and performance analysis," IEEE Journal on Selected Areas in Communications, vol. 12, no. 3, pp. 433-438, April 1994.

[15] V. A. Bolotin, "Telephone circuit holding time distributions," in Proc. of $14^{\text {th }}$ International Teletraffic Congress (ITC'94), June 1994, pp. 125-134.

[16] J. Jordán and F. Barceló, "Statistical modelling of channel occupancy in PAMR systems," in Proc. of $15^{\text {th }}$ International Teletraffic Congress (ITC'97), June 1997, pp. 1169-1178.

[17] F. Barceló and J. Jordán, "Voice holding time distribution in trunked PAMR systems," in Proc. of IEEE Vehicular Technology Conference (VTC'97), May 1997, pp. 436-440.

[18] J. Jordán and F. Barceló, "Statistical modelling of transmission holding time in PAMR systems," in Proc. of IEEE Global Telecommunications Conference (GLOBECOM '97), Nov. 1997, vol. 1, pp. 121-125.

[19] F. Barceló and J. Jordán, "Channel holding time distribution in public telephony systems (PAMR and PCS)," IEEE Trans. on Vehicular Technology, vol. 49, no. 5, pp. 1615-1625, Sept. 2000.

[20] C. Jedrzycki and V. Leung, "Probability distribution of channel holding time in cellular telephony systems," in Proc. of IEEE $46^{\text {th }}$ Vehicular Technology Conference (VTC'96), April 1996, vol. 1, pp. 247-251.

[21] P. V. Orlik and S. S. Rappaport, "Traffic performance and mobility modeling of cellular communications with mixed platforms and highly variable mobilities," Proc. of the IEEE, vol. 86, no. 7, pp. 1464-1479, July 1998.

[22] P. V. Orlik and S. S. Rappaport, "A model for teletraffic performance and channel holding time characterization in wireless cellular communication with general session and dwell time distributions," IEEE Journal on Selected Areas in Communications, vol. 16, no. 5, pp. 788-803, June 1998.

[23] Y. Fang, "Hyper-Erlang distribution model and its application in wireless mobile networks," Wireless Networks (WINET), vol.7, no.3, pp. 211-219, May 2001.

[24] Y. Fang and I. Chlamtac, "Teletraffic analysis and mobility modeling of PCS networks," IEEE Trans. on Communications, vol. 47, no. 7, pp. 1062-1072, July 1999.

[25] Y. Fang, I. Chlamtac, and Y. B. Lin, "Channel occupancy times and handoff rate for mobile computing and PCS networks," IEEE Trans. on Computers, vol. 47, no. 6, pp. 679-692, June 1998.

[26] Y. Lin and I. Chlamtac, "Effects of Erlang call holding times on PCS call completion," IEEE Transactions on Vehicular Technology, vol. 48, no. 3, pp. 815-823, May 1999.

[27] D. Sharp, N. Cackov, N. Lasković, Q. Shao, and Lj. Trajković, "Analysis of public safety traffic on trunked land mobile radio systems," IEEE Journal on Selected Areas in Communications, vol. 22, no. 7, pp. 1197-1205, Sept. 2004. 
[28] V. Frost and B. Melamed, "Traffic modeling for telecommunications networks," IEEE Communications Magazine, vol. 32, no. 3, pp. 70-80, Mar. 1994.

[29] F. Barceló and S. Bueno, "Idle and inter-arrival time statistics in public access mobile radio (PAMR) systems," in IEEE Global Telecommunications Conference (GLOBECOM'97), Nov. 1997, vol. 1, pp. 126-130.

[30] F. Barceló and J. I. Sánchez, "Probability distribution of the inter-arrival time to cellular telephony channels," in Proc. of the $49^{\text {th }}$ Vehicular Technology Conference (VTC'99), May 1999, vol. 1, pp. 762-766.

[31] M. Rajaratnam and F. Takawira, "Handoff traffic characterization in cellular networks under nonclassical arrivals and service time distributions," IEEE Trans. on Vehicular Technology, vol. 50, no. 4, pp. 954-970, July 2001.

[32] OPNET Modeler [Online]. Available: http://www.opnet.com.

[33] ns-2 [Online]. Available: http://www.isi.edu/nsnam/ns.

[34] J-Sim [Online]. Available: http://www.j-sim.org.

[35] WarnSim [Online]. Available: http://www.vannet.ca/warnsim.

[36] J. Song and Lj. Trajković, "Modeling and performance analysis of public safety wireless networks," in Proc. of the $24^{\text {th }}$ IEEE International Performance, Computing, and Communication Conference (IPCCC'05), April 2005, pp. 567572.

[37] ToMMTi-Systems. Performance comparison C++, C\# and Java [Online]. Available: http://www.tommti-systems.de/main-Dateien/reviews/languages/ benchmarks.html. Accessed: Aug. 15, 2004.

[38] M. Matsumoto and T. Nishimura, "Mersenne twister: a 623-dimensionally equidistributed uniform pseudorandom number generator," ACM Transactions on Modeling and Computer Simulation, vol. 8, no. 1, pp. 3-30, Jan. 1998.

[39] MATLAB [Online]. Available: http://www.mathworks.com/products/matlab.

[40] S-PLUS [Online]. Available: http://www.insightful.com/products/splus.

[41] H. Chen and Lj. Trajković, "Trunked radio systems: traffic prediction based on user clusters," in Proc. of International Symposium on Wireless Communication Systems 2004, Sept. 2004, pp. 76-80.

[42] D. Sharp, "Adapting ad hoc network concepts to land mobile radio systems," M. Eng. project, School of Engineering Science, Simon Fraser Univ., Burnaby, BC, Canada, 2002. 